\title{
BOUNDED ANALYTIC FUNCTIONS ON DOMAINS OF INFINITE CONNECTIVITY
}

\author{
BY \\ LAWRENCE ZALCMAN( $\left.{ }^{1}\right)$
}

1. Introduction. This paper is a contribution to the study of bounded analytic functions on plane domains of infinite connectivity. For a fixed domain $D$, the set $B(D)$ of all such functions is a Banach algebra under the natural (pointwise) operations and the uniform norm. Thus, our results generalize theorems about $H^{\infty}$, the much-studied Banach algebra of all functions bounded and analytic on the open unit disc.

The plan of the paper is this. $\$ 2$ is devoted to a description of the kind of domains we shall consider and to various preliminaries concerning the representation and approximation of functions on these domains. In $\S 3$, we prove that the algebra $A(\bar{D})$ of all functions continuous on $\overline{\bar{D}}$ and analytic on $D$ is weak $*$ dense in $B(D)$. We conclude, in passing, that for each $f$ in $B(D)$ there exists a sequence $f_{n}$ of functions in $A(\bar{D})$ such that $\left\|f_{n}\right\|_{\infty} \leqq\|f\|_{\infty}$ and $f_{n}(z) \rightarrow f(z)$ uniformly on compact subsets of $D$; in fact, $f_{n} \rightarrow f$ almost everywhere on $\partial D$. $\S 4$ brings together some facts which relate the boundary behavior of functions in $B(D)$ to the geometry of the maximal ideal space $M$ of $B(D)$. $\S 5$ focuses attention on a special homomorphism in $M$ which has several interesting properties. In $\S 6$, we are concerned with extending a recent theorem of Sarason from the disc to our more general domains. It was this problem that originally captured our interest and provided much of the motivation for the research in this paper. The purpose of including these somewhat more specialized results here is to point up some of the problems that arise in studying infinitely connected domains and to indicate how these difficulties may be circumvented. $\$ 7$ consists of comments on the generality of our arguments and explains how various results can be extended to a larger class of domains. Finally, in $\S 8$, we offer a brief survey of the literature, together with some comments on the points of tangency between our work and that of other mathematicians.

Throughout, we shall assume that the reader is familiar with the basic $H^{\infty}$ terminology and theory as presented in [17].

This paper is the author's doctoral thesis, submitted to the Massachusetts Institute of Technology. I should like to thank Professor Kenneth Hoffman, who directed this work, for his patience and encouragement. I also wish to thank the referee for his many helpful suggestions.

Received by the editors May 27, 1968 and, in revised form, August 8, 1968.

( $\left.{ }^{1}\right)$ Part of this research was carried out while the author held a National Science Foundation Graduate Fellowship. Research at Stanford supported by contract AF 49(638)1345. 
2. Preliminaries. We shall be concerned with $B(D)$ for certain sets $D$ whose topological structure is especially simple. To be precise, $D$ will always denote a domain obtained by deleting from the punctured disc $\{0<|z|<1\}$ a disjoint sequence of closed discs $\left\{\left|z-x_{n}\right| \leqq r_{n}\right\}$ centered on the positive real axis and accumulating only at 0 . By analogy with the terminology for compact sets [29, p. 57], we call $D$ a domain of type $(L)$. Such a domain is obviously the interior of its closure $\left(D=(\bar{D})^{0}\right)$, and moreover, its boundary $\partial D$ has finite length. As usual, we denote arc length measure on $\partial D$ by $d s$.

We shall find the following notation convenient: $\Delta=\{|z|<1\}, \gamma_{0}=\{|z|=1\}$ $\gamma_{n}=\left\{\left|z-x_{n}\right|=r_{n}\right\}(n=1,2, \ldots), \Gamma_{n}=\bigcup_{k=0}^{n} \gamma_{k}, \Gamma=\partial D$. For purposes of integration, we orient $\gamma_{0}$ in the counterclockwise direction and $\gamma_{n}(n \geqq 1)$ in the clockwise sense and endow $\Gamma_{n}(n=0,1,2, \ldots)$ with the corresponding orientation. Unless otherwise specified, $R$ is an arbitrary plane domain. Interior points of $R$ (or $D$ ) will be denoted by italic letters (most often $z$ ), while the generic boundary point will be called $\zeta$ or $\xi$.

At this point it seems worth remarking that it is principally for reasons of convenience that we have stated our theorems in terms of domains of type $(L)$ : virtually every result will have more-or-less obvious extensions to more general sorts of domains. Frequently we shall note these extensions explicitly; less obvious generalizations will be treated in $\S 7$.

Now suppose $D$ is given. Then we have

Proposition 2.1. Let $f \in B(D)$. Then $f$ has nontangential boundary values at almost every point of $\partial D$.

Proof. This is an immediate consequence of the corresponding fact for $H^{\infty}$ functions. Indeed, if $\zeta \in \partial D \backslash\{0\}$ then $\zeta$ has a neighborhood $N$ in $\bar{D}$ whose interior is analytically equivalent to $\Delta$; moreover, $\zeta$ lies on an analytic subarc of $\partial N$. If $\phi$ maps $\Delta$ conformally onto $N$, then $f \circ \phi \in H^{\infty}$; and $\phi$ extends analytically to a full neighborhood of $\phi^{-1}(\zeta)$ by the reflection principle. Hence, a nontangential path at $\phi^{-1}(\zeta)$ is mapped onto a nontangential path at $\zeta$. It follows that $f$ has nontangential boundary values almost everywhere $(d \theta)$ on each $\gamma_{n}$. Since $\partial D \backslash \bigcup \gamma_{n}$ $=\{0\}$ has arclength 0 and $d s \ll d \theta$ on each $\gamma_{n}$, these boundary values exist almost everywhere on $\partial D(d s)$.

We denote the boundary function of $f(z) \in B(D)$ by $f(\zeta)$ and observe that $f(\zeta)$ is a bounded measurable function on $\Gamma=\partial D$. The interior function $f(z)$ and the boundary function $f(\zeta)$ are related by

Proposition 2.2. Let $f(z) \in B(D)$. Then

$$
f(z)=\frac{1}{2 \pi i} \int_{\Gamma} \frac{f(\zeta)}{\zeta-z} d \zeta
$$


Proof. Fix $z$ and let $C_{n}=\left\{|z|=\varepsilon_{n}\right\}$ be a counterclockwise oriented circle in $D$ which separates $\gamma_{n}$ from $\gamma_{n+1}$. By Cauchy's theorem, we have

$$
f(z)=\frac{1}{2 \pi i} \int_{\Gamma_{n}} \frac{f(\zeta)}{\zeta-z} d \zeta-\frac{1}{2 \pi i} \int_{C_{n}} \frac{f(w)}{w-z} d w=I_{1}(n)+I_{2}(n)
$$

for large enough $n$. The integral $I_{2}(n)$ is bounded by $\|f\|_{\infty} \varepsilon_{n}\left[d\left(z, C_{n}\right)\right]^{-1}$, where $d\left(z, C_{n}\right)$ is the distance from $z$ to $C_{n}$. Thus as $n \rightarrow \infty, I_{2}(n) \rightarrow 0$. If we take limits on both sides, we obtain the desired conclusion.

Proposition 2.2 remains valid for domains whose (rectifiable) boundary consists of a countable number of disjoint smooth simple closed curves $\tau_{n}$ which accumulate on a (totally disconnected) set $S$ of Hausdorff linear measure 0. ( $S$ may, but need not, intersect $\bigcup \tau_{n}$.) Indeed, in this case, each bounded analytic function has appropriately defined boundary values (cf. $\S 7$ ), and the proof of 2.2 can be applied with only slight modification. It seems likely that the Hausdorff measure condition can be weakened to the requirement that $S$ have zero analytic capacity [29].

Generally speaking, however, the representation of 2.2 fails utterly. Of course, if $\partial R$ is not rectifiable, the boundary function may not exist in the proper sense; and, if it does exist, the integral will still fail to converge absolutely. Moreover, if $\partial R$ is rectifiable or even analytic 2.2 may break down if the set at which the boundary curves accumulate is too large. For instance, we have

ExAmple 2.3. Let $S$ be the slit $[-i / 2, i / 2]$, and let $R$ be the region obtained by deleting from the slit disc $\Delta \mid S$ a disjoint sequence $\bar{\Delta}_{n}(n=1,2, \ldots)$ of closed discs with radii $r_{n}$ such that $\sum r_{n}<\infty$ and the $\bar{\Delta}_{n}$ accumulate at every point of $S$ (on both sides) and at no other points. Let $\tau_{n}=\partial \Delta_{n}$ (oriented clockwise), $\tau_{0}=\gamma_{0}$. If $f \in B(R)$, then $f$ has nontangential boundary values at almost every ( $d s)$ point of $\bigcup \tau_{n}$. However, in general, $f$ will not have well-defined boundary values at points of $S$. We claim that there exists $f \in B(R)$ such that

$$
f(z) \neq \frac{1}{2 \pi i} \int_{\bigcup_{n=0}^{\infty} \tau_{n}} \frac{f(\zeta)}{\zeta-z} d \zeta
$$

Indeed, let $f$ be a Riemann map of the complement of $S$ in the complex sphere onto $\Delta$; then clearly $f \in B(R)$. Also,

$$
\int_{\tau_{n}} f(\zeta) d \zeta=0, \quad n=1,2, \ldots
$$

since $f$ is analytic in a disc containing each $\tau_{n}$. Now suppose

$$
f(z)=\frac{1}{2 \pi i} \int_{\bigcup_{n=0}^{\infty} \tau_{n}} \frac{f(\zeta)}{\zeta-z} d \zeta
$$

and let $C \subset R$ be a smooth curve which surrounds $S$ once. We have

$$
\int_{C} f(z) d z=2 \pi i f^{\prime}(\infty) \neq 0
$$


where the integral is taken in the counterclockwise direction and $f^{\prime}(\infty)$ is the coefficient of $z^{-1}$ in the expansion of $f$ at $\infty$; this coefficient is nonzero since $f$ is schlicht in the complement of $S$. However,

$$
\int_{C}\left(\int_{U \tau_{n}} \frac{f(\zeta)}{\zeta-z} d \zeta\right) d z=\int_{U \tau_{n}} f(\zeta)\left(-\int_{C} \frac{d z}{z-\zeta}\right) d \zeta
$$

Since

$$
\begin{aligned}
\frac{1}{2 \pi i} \int_{C} \frac{d z}{z-\zeta} & =1 & & \text { if } \zeta \text { lies inside } C \\
& =0 & & \text { otherwise }
\end{aligned}
$$

it follows from (2) and (6) that the right-hand side of (5) above is 0 , contradicting the conjunction of (3) and (4). Hence (1) holds, as was claimed.

The informed reader will have noted that what is of relevance to the above construction is that $S$ has positive analytic capacity; $S$ could have been replaced by any closed set of positive analytic capacity. For instance, one could have chosen any compact subset of the segment $(-i, i)$ of positive linear measure.

REMARK. In 2.3 above it is not hard to see that if $g \in A(\bar{R})$ one still has

$$
g(z)=\frac{1}{2 \pi i} \int_{\cup \tau_{n}} \frac{g(\zeta)}{\zeta-z} d \zeta
$$

However, in general, even this sort of representation will fail; one need only choose the set at which the "nice" boundary accumulates to be sufficiently thick. For instance, let $R$ be a region whose boundary consists of a union of circles $\tau_{n}$ of finite total length plus a set of positive $A C$ capacity [29, p. 77] (e.g., an arc of positive two-dimensional measure) at which the circles accumulate. Then it is not true that each function in $A(\bar{R})$ can be represented as the Cauchy integral of its (continuous!) boundary function taken over $\bigcup \tau_{n}$ (cf. [29, pp. 69-76]). On the other hand, in such cases $\partial R$ is not rectifiable. Indeed, it is easy to show that if a set has finite Hausdorff linear measure it has zero $A C$ capacity.

We shall also need the following result from the theory of rational approximation.

THEOREM 2.4. Let $D$ be a domain of type $(L)$. Each function in $A(\bar{D})$ can be approximated uniformly on $\bar{D}$ by rational functions with poles off $\bar{D}$. These poles may be chosen to lie in the set $\left\{\infty, x_{1}, x_{2}, \ldots\right\}$.

Proof. Let $g_{n}$ be a $C^{\infty}$ function defined on the plane such that

(1) $g_{n}=1$ on $\{|z| \leqq 1 / n\}$,

(2) $g_{n}=0$ on $\{|z| \geqq 2 / n\}$,

(3) $\left\|\partial g_{n} / \partial \bar{z}\right\|_{\infty} \leqq 10 n$.

Suppose $f \in A(\bar{D})$, and let $\varepsilon>0$ be given. Extend $f$ continuously to a (bounded) function on the complex sphere and set

$$
\begin{aligned}
f_{n}(z) & =\frac{1}{\pi} \iint \frac{f(w)-f(z)}{w-z} \frac{\partial g_{n}}{\partial \bar{w}} d x d y \\
& =f(z) g_{n}(z)+\frac{1}{\pi} \iint \frac{f(w)}{w-z} \frac{\partial g_{n}}{\partial \bar{w}} d x d y, \quad w=x+i y .
\end{aligned}
$$


(Here $z$ is not necessarily restricted to $D$ and the integration is taken over the entire complex plane.) It is easy to see (cf. [29, pp. 88-89]) that $f_{n}$ is continuous on the whole plane and analytic on $D$; moreover, $\left\|f_{n}\right\|_{\infty} \leqq 40 \omega_{f}(4 / n)$, where $\omega_{f}(\delta)$ is the modulus of continuity of (the extension of) $f$. Since $f$ is uniformly continuous, $\left\|f_{n}\right\|_{\infty} \rightarrow 0$. Further, $f-f_{n}$ is analytic on $\{|z| \leqq 1 / n\}$ since the measure $\left(\partial g_{n} / \partial \bar{w}\right) d x d y$ has no mass there. Thus $h_{n}=f-f_{n}$ is continuous on $\bar{D}$ and analytic on $D$ as well as a full neighborhood of 0 ; i.e., for large enough $k=k(n), h_{n} \in A\left(\bar{D}_{k}\right)$, where $D_{k}$ is the finitely connected domain bounded by $\Gamma_{k}$. By a classical theorem, each function in $A\left(\bar{D}_{k}\right)$ can be approximated uniformly by rational functions whose poles lie in $\left\{\infty, x_{1}, x_{2}, \ldots, x_{k}\right\}$. Choose $n$ so large that $\omega_{f}(4 / n)<\varepsilon / 80$ and then pick a rational function $r$ with poles in $\left\{\infty, x_{1}, x_{2}, \ldots, x_{k}\right\}$ such that $\left\|h_{n}-r\right\|_{D_{k}}<\varepsilon / 2$. We have

as required.

$$
\|f-r\|_{\bar{D}} \leqq\left\|h_{n}-r\right\|_{D_{k}}+\left\|f_{n}\right\|_{\bar{D}}<\varepsilon
$$

The preceding proof is T. W. Gamelin's rearrangement of an argument due to John Garnett (cf. [29, pp. 126-128]). Actually, 2.4 is a special case of a very general theorem of A. G. Vitushkin; it is only for completeness and for the reader's convenience that we have included it here. Since we have treated these matters in considerable detail elsewhere [29], we shall not comment on them further.

Finally, we require a result from the theory of cluster sets [6]. Recall that if $f$ is an (arbitrary) function defined on a plane domain $R$ the cluster set of $f$ at $\alpha \in \bar{R}$, denoted by $\mathrm{Cl}(f ; \alpha)$, is the set of limiting values of $f$ at $\alpha$. More precisely, $\beta \in \mathrm{Cl}(f ; \alpha)$ if and only if there exists a sequence $z_{n} \in R \mid\{\alpha\}$ such that $z_{n} \rightarrow \alpha$ and $f\left(z_{n}\right) \rightarrow \beta$. If $\alpha$ is a nonisolated boundary point of $R$, one can also define the boundary cluster set of $f$ at $\alpha, \mathrm{Cl}_{\mathrm{B}}(f ; \alpha)$. We write $\beta \in \mathrm{Cl}_{\mathrm{B}}(f ; \alpha)$ if and only if there exists a sequence $\left\{\alpha_{n}\right\} \subset \partial R \backslash\{\alpha\}$ and elements $\beta_{n} \in \mathrm{Cl}\left(f ; \alpha_{n}\right)$ such that $\alpha_{n} \rightarrow \alpha$, $\beta_{n} \rightarrow \beta$. In $1914 \mathrm{~F}$. Iversen proved the following beautiful and important theorem.

THEOREM 2.5. Let $R$ be a bounded domain and let $\zeta \in \partial R$ be a nonisolated boundary point. Then if $f$ is meromorphic on $R$

$$
\partial(\mathrm{Cl}(f ; \zeta)) \subset \mathrm{Cl}_{\mathrm{B}}(f ; \zeta)
$$

Proof. [26, pp. 332-335].

Of course, we shall be interested in the case $R=D, f \in B(D)$.

3. Weak star density. According to 2.1 and 2.2, the correspondence between bounded analytic functions on $D$ and their boundary-value functions is an isomorphism between $B(D)$ and a subalgebra of $L^{\infty}(\partial D ; d s)$. There are various ways of seeing that this isomorphism is actually an isometry. For instance, we may observe that, by 2.2 , evaluation at $z \in D$ is a continuous homomorphism of the boundary value algebra onto the complex numbers and hence extends to the $L^{\infty}$ closure of this algebra. Since this latter algebra is complete, the homomorphism must actually have unit norm. Letting $z$ vary over $D$, we obtain the required conclusion. It is now obvious that the boundary algebra was actually closed to 
begin with. We shall identify $B(D)$ with its (closed) boundary-value algebra and, by an abuse of language, denote the latter algebra also by $B(D)$. A similar convention will hold for $A(\bar{D})$, which we regard as a closed subalgebra of $B(D)$. Thus, in the sequel, context will determine whether functions of $B(D)$ or $A(\bar{D})$ are to be regarded as defined on $D$ or on $\partial D$.

By the weak * topology on $B(D)$ we mean the weak topology $B(D)$ inherits as a closed subspace of $L^{\infty}(\partial D ; d s)$ in the pairing $\left(L^{\infty}(d s), L^{1}(d s)\right)$. The principal result of this section is

THEOREM 3.1. $B(D)$ is the weak * closure of $A(\bar{D})$ in $L^{\infty}(\partial D ; d s)$.

The proof will proceed by a series of auxiliary propositions. First of all, we have LEMMA 3.2. Let $f \in L^{1}(\partial D ; d s)$ satisfy

$$
\int_{\Gamma} f(\zeta) g(\zeta) d \zeta=0
$$

for all $g \in A(\bar{D})$ and set

$$
f(z)=\frac{1}{2 \pi i} \int_{\Gamma} \frac{f(\zeta)}{\zeta-z} d \zeta, \quad z \in D
$$

Then, for almost every $\zeta \in \partial D, f(z) \rightarrow f(\zeta)$ as $z \rightarrow \zeta$ nontangentially. Moreover,

$$
\begin{gathered}
\lim _{r \rightarrow 1-} \int_{0}^{2 \pi}\left|f\left(e^{i \theta}\right)-f\left(r e^{i \theta}\right)\right| d \theta=0 \\
\lim _{r \rightarrow r_{n}+} \int_{0}^{2 \pi}\left|f\left(x_{n}+r_{n} e^{i \theta}\right)-f\left(x_{n}+r e^{i \theta}\right)\right| d \theta=0 \quad(n=1,2, \ldots) .
\end{gathered}
$$

Proof. Let, as in $2.4, D_{n}$ be the (finitely-connected) domain bounded by $\Gamma_{n}$ $(n=1,2, \ldots)$ and set, for $\zeta \in \Gamma_{n}$,

$$
f_{n}(\zeta)=f(\zeta)-\frac{1}{2 \pi i} \int_{\Gamma \mid \Gamma_{n}} \frac{f(\xi)}{\xi-\zeta} d \xi
$$

Suppose $g \in A\left(\bar{D}_{n}\right)$. Then if $\nu$ is a finite measure on $\Gamma \mid \Gamma_{n}$ we have the following balayage formula:

$$
\begin{aligned}
\int_{\Gamma \mid \Gamma_{n}} g(\xi) d \nu(\xi) & =\int_{\Gamma \mid \Gamma_{n}}\left(\frac{1}{2 \pi i} \int_{\Gamma_{n}} g(\zeta) \frac{d \zeta}{\zeta-\xi}\right) d \nu(\xi) \\
& =\int_{\Gamma_{n}} g(\zeta)\left(\frac{1}{2 \pi i} \int_{\Gamma \mid \Gamma_{n}} \frac{d \nu(\xi)}{\zeta-\xi}\right) d \zeta .
\end{aligned}
$$

We shall choose $d \nu(\xi)=f(\xi) d \xi$. Since $g \in A(\bar{D})$ we have by (1)

$$
0=\int_{\Gamma} g(\xi) f(\xi) d \xi=\int_{\Gamma_{n}}+\int_{\Gamma \mid \Gamma_{n}} .
$$


Hence, by (5) and (6),

$$
\begin{aligned}
\int_{\Gamma_{n}} g(\xi) f(\xi) d \xi & =-\int_{\Gamma_{n}} g(\zeta)\left(\frac{1}{2 \pi i} \int_{\Gamma \mid \Gamma_{n}} \frac{f(\xi)}{\zeta-\xi} d \xi\right) d \zeta \\
& =\int_{\Gamma_{n}} g(\zeta)\left(\frac{1}{2 \pi i} \int_{\Gamma \mid \Gamma_{n}} \frac{f(\xi)}{\xi-\zeta} d \xi\right) d \zeta
\end{aligned}
$$

Combining (4) and (7) we get

$$
\int_{\Gamma_{n}} g(\zeta) f_{n}(\zeta) d \zeta=0, \quad g \in A\left(\bar{D}_{n}\right)
$$

Thus $f_{n}(\zeta) \in H^{1}\left(D_{n}\right)$ [22]. Therefore, for $z \in D_{n}$,

$$
\begin{aligned}
f_{n}(z) & =\frac{1}{2 \pi i} \int_{\Gamma_{n}} \frac{f_{n}(\zeta)}{\zeta-z} d \zeta \\
& =\frac{1}{2 \pi i} \int_{\Gamma_{n}} \frac{f(\zeta)}{\zeta-z} d \zeta-\frac{1}{2 \pi i} \int_{\Gamma_{n}}\left(\frac{1}{2 \pi i} \int_{\Gamma \mid \Gamma_{n}} \frac{f(\xi)}{\xi-\zeta} d \xi\right) \frac{d \zeta}{\zeta-z} \\
& =\frac{1}{2 \pi i} \int_{\Gamma_{n}} \frac{f(\zeta)}{\zeta-z} d \zeta-\left(\frac{1}{2 \pi i}\right)^{2} \int_{\Gamma \mid \Gamma_{n}}\left(\int_{\Gamma_{n}} \frac{d \zeta}{(\zeta-z)(\xi-\zeta)}\right) f(\xi) d \xi
\end{aligned}
$$

A partial fraction decomposition and the residue theorem show that the last integral in (9) vanishes. Thus

$$
f_{n}(z)=\frac{1}{2 \pi i} \int_{\Gamma_{n}} \frac{f(\zeta)}{\zeta-z} d \zeta
$$

and so

$$
f(z)=f_{n}(z)+\frac{1}{2 \pi i} \int_{\Gamma \mid \Gamma_{n}} \frac{f(\xi)}{\xi-z} d \xi .
$$

Since $f_{n} \in H^{1}\left(D_{n}\right), f_{n}(z) \rightarrow f_{n}(\zeta)$ a.e. on $\Gamma_{n}$ as $z \rightarrow \zeta \in \Gamma_{n}$ nontangentially [22]. Thus, for a.a. $\zeta \in \Gamma_{n}$

$$
\begin{aligned}
\lim _{z \rightarrow \zeta} f(z) & =\lim _{z \rightarrow \zeta} f_{n}(z)+\lim _{z \rightarrow \zeta} \frac{1}{2 \pi i} \int_{\Gamma \mid \Gamma_{n}} \frac{f(\xi)}{\xi-z} d \xi \\
& =f_{n}(\zeta)+\frac{1}{2 \pi i} \int_{\Gamma \mid \Gamma_{n}} \frac{f(\xi)}{\xi-\zeta} d \xi=f(\zeta)
\end{aligned}
$$

for nontangential approach.

In a similar way, the second part of the lemma follows from the representation (11).

A similar result, with a different proof, is given in [9].

Next we have

Proposition 3.3. Suppose $h \in B(D)$ is continuous on $\bar{D} \mid\{0\}$. Then $h$ can be approximated pointwise boundedly on $\partial D \mid\{0\}$ by functions in $A(\bar{D})$. 
Proof. The proof uses a technique due to Arens [2, pp. 644-646]. Let $H(z)=z h(z)$; then $H \in A(\bar{D})$. Let $H(z)=0, z \notin \bar{D}$, and write

$$
h_{n}(z)=\frac{n^{2}}{\pi} \iint_{S_{n}} \frac{H(w)-H(z)}{w-z} d x d y, \quad w=x+i y,
$$

where $S_{n}=\{|w| \leqq 1 / n\}$. Here $z$ is allowed to vary over the whole plane. Then $h_{n}$, being (essentially) the sum of convolutions, is continuous on $\bar{D}$; moreover, it is not hard to see that it is analytic on $D\left[2\right.$, p. 644]. Thus $h_{n}(z) \in A(\bar{D})$. Now

$$
z h_{n}(z)-H(z)=\frac{n^{2}}{\pi} \iint_{S_{n}} \frac{z H(w)-w H(z)}{w-z} d x d y
$$

so

$$
\left|z h_{n}(z)-H(z)\right| \leqq(|z| b(1 / n)+(1 / n)|H(z)|)\left(\frac{n^{2}}{\pi} \iint_{S_{n}} \frac{d x d y}{|w-z|}\right)
$$

where $b(\delta)=\sup \{|H(z)|:|z|<\delta\}$. It follows that

or

$$
\left|z h_{n}(z)-H(z)\right| \leqq(|z| b(1 / n)+(1 / n)|z||h(z)|) 2 n
$$

$$
\left|h_{n}(z)-h(z)\right| \leqq 2 n b(1 / n)+2|h(z)| .
$$

Now $b(1 / n)=\sup \left\{|z h(z)|: z \in S_{n}\right\} \leqq(1 / n)\|h\|_{\infty}$. Thus

$$
\left|h_{n}(z)-h(z)\right| \leqq 4\|h\|_{\infty}
$$

so that $\left\|h_{n}\right\|_{\infty} \leqq 5\|h\|_{\infty}$. It is shown in [2] that $z h_{n} \rightarrow H$ on $\bar{D}$ uniformly. It follows that $h_{n} \rightarrow h$ pointwise boundedly on $\bar{D} \backslash\{0\}$ and that convergence is uniform off any neighborhood of 0 . That completes the proof.

A different proof of 3.3 can be based on the technique of 2.4 .

Finally, we need the following general result [19, p. 454].

Proposition 3.4. Let $\mu$ be a finite positive measure and let $S$ be a subspace of $L^{\infty}(d \mu) . S$ is weak ${ }^{*}$ closed if and only if $\left\{f_{n}\right\} \subset S, f_{n} \rightarrow f$ pointwise boundedly a.e. implies that $f \in S$.

Proof. One direction is clear. Suppose that each boundedly pointwise convergent sequence in $S$ converges to an element of $S$. We claim $S$ is weak * closed. It is enough, by the Krein-Shmulian theorem [7, p. 429], to show that the unit ball $U$ of $S$ is (weak ${ }^{*}$ ) closed. If $f$ is in the weak * closure of $U$ it is also in the weak $L^{2}$ closure of $U$ and hence in the (norm) $L^{2}$ closure of $U$, since $U$ is convex. It follows that some sequence in $U$ converges to $f$ in $L^{2}(d \mu)$, so an appropriate subsequence converges pointwise a.e. $(d \mu)$. By hypothesis, $f \in S$.

Actually, 3.4 holds for arbitrary convex sets in $L^{\infty}$; the proof remains unchanged.

Proof of 3.1. First we shall prove that $A(\bar{D})$ is weak * dense in $B(D)$. It is, of course, enough to show that any function $f \in L^{1}(\partial D ; d s)$ which is orthogonal to $A(\bar{D})$ is also orthogonal to $B(D)$. Suppose, then, that

$$
\int_{\Gamma} f(\zeta) g(\zeta)=0, \quad g \in A(\bar{D})
$$


Choose curves $\gamma_{k}^{\prime}(k=0,1,2, \ldots)$ in $D$ concentric with $\gamma_{k}$ in such a way that $\gamma_{k}^{\prime}$ separates $\gamma_{k}$ from $\Gamma \mid \gamma_{k}$ and let $D^{\prime}$ denote the subdomain of $D$ bounded by the $\gamma_{k}^{\prime}$, $\Gamma^{\prime}=\partial D^{\prime} . D^{\prime}$ is clearly a domain of type $(L)$. Further, since by 3.2 the analytic extension (Cauchy transform) $f(z)$ of $f$ converges back to $f$ in $L^{1}$ norm on each boundary curve $\gamma_{k}$, we may assume that the curves $\gamma_{k}^{\prime}$ have been chosen in such a way that $f(z) \in L^{1}\left(\partial D^{\prime} ; d s\right)$.

Now let $h \in B(D)$ be fixed. We must show

$$
\int_{\Gamma} f(\zeta) h(\zeta) d \zeta=0
$$

For each $k$ we have (by 3.2 and the Cauchy theorem)

$$
\int_{\gamma_{k}} f(\zeta) h(\zeta) d \zeta=\int_{\gamma_{k}^{\prime}} f(z) h(z) d z
$$

Hence, since all integrals are absolutely convergent,

$$
\int_{\Gamma} f(\zeta) h(\zeta) d \zeta=\int_{\Gamma^{\prime}} f(z) h(z) d z .
$$

By (1) and (4),

$$
\int_{\Gamma^{\prime}} f(z) r(z) d z=0
$$

for each rational function $r$ with poles off $\bar{D}$. It follows, by 2.4 , that

$$
\int_{\Gamma^{\prime}} f(z) g(z) d z=0, \quad g \in A\left(\bar{D}^{\prime}\right) .
$$

Now let $h_{n}$ be as in 3.3. Then $h_{n} \in A\left(\bar{D}^{\prime}\right)$ and $h_{n}(z) \rightarrow h(z)$ pointwise boundedly on $\partial D^{\prime} \backslash\{0\}$. Hence, by (6), we have

$$
0=\lim _{n} \int_{\Gamma^{\prime}} f(z) h_{n}(z) d z=\int_{\Gamma^{\prime}} f(z) h(z) d z .
$$

Combining (4) and (7), we obtain (2), as required.

Since $A(\bar{D}) \subset B(D)$, it remains only to show that $B(D)$ is weak * closed. We shall verify the condition of 3.4. Suppose, then, that $f_{n} \rightarrow f$ pointwise boundedly a.e. on $\partial D, f_{n} \in B(D)$. Since $f_{n}$ is a normal family, there exists $g \in B(D)$ such that $f_{n}(z) \rightarrow g(z)$ uniformly on compact subsets of $D$. Clearly,

$$
g(z)=\frac{1}{2 \pi i} \int_{\Gamma} \frac{f(\zeta)}{\zeta-z} d \zeta
$$

Hence

$$
\frac{1}{2 \pi i} \int_{\Gamma} \frac{f(\zeta)-g(\zeta)}{\zeta-z} d \zeta=0, \quad z \in D
$$

On the other hand, by Cauchy's theorem,

$$
\frac{1}{2 \pi i} \int_{\Gamma} \frac{h(\zeta)}{\zeta-z} d \zeta=0, \quad h \in B(D)
$$


if $z \notin \bar{D}$. Thus, by the dominated convergence theorem,

$$
\frac{1}{2 \pi i} \int_{\Gamma} \frac{f(\zeta)-g(\zeta)}{\zeta-z} d \zeta=0
$$

for $z \notin \bar{D}$. By (9) and (11)

$$
\int_{\Gamma}(f(\zeta)-g(\zeta)) \frac{1}{\zeta-z} d \zeta=0
$$

for any $z \notin \partial D$. It follows by the theorem of Hartogs and Rosenthal $[29$, p. 110] that

$$
\int_{\Gamma}(f(\zeta)-g(\zeta)) k(\zeta) d \zeta=0
$$

for any $k \in C(\partial D)$. Evidently, $f(\zeta)=g(\zeta)$ a.e. on $\partial D$, i.e., $f \in B(D)$. The proof is complete.

COROLlaRY 3.5. If $\mu$ is a positive measure on $\partial D$ which is absolutely continuous with respect to ds then $B(D)$ is the weak * closure of $A(\bar{D})$ in $L^{\infty}(d \mu)$.

Proof. Obvious.

In particular, if $z \in D$ is fixed and $\mu_{z}$ denotes harmonic measure for $z$, we have $d \mu_{z} \ll d s$, so that 3.5 applies.

As an application of 3.1 we have

THEOREM 3.6. Given $f \in B(D)$, there exists a sequence $\left\{f_{n}\right\}$ of functions in $A(D)$ such that

$$
\begin{gathered}
\left\|f_{n}\right\|_{\infty} \leqq\|f\|_{\infty}, \\
f_{n}(\zeta) \rightarrow f(\zeta) \text { a.e. on } \partial D .
\end{gathered}
$$

In particular, $f_{n}(z) \rightarrow f(z)$ for each $z \in D$, and the convergence is uniform on compact subsets of $D$.

Proof. By [1, p. 939] it is enough to prove that

$$
\sup \left\{\left|\int h f d s\right|: h \in B(D),\|h\|_{\infty} \leqq 1\right\} \leqq \sup \left\{\left|\int g f d s\right|: g \in A(\bar{D}),\|g\|_{\infty} \leqq 1\right\}
$$

for each $f \in L^{1}(d s)$. In other words, we must show that the functional which $f$ induces on $B(D)$ has the same norm as that of the functional which $f$ induces on $A(\bar{D})$. Denote the norm of the latter functional by $N$. By the Hahn-Banach theorem and the Riesz representation theorem, there exists a measure $\mu$ on $\partial D$ such that $\int g d \mu=\int g f d s$ for all $g \in A(\bar{D})$, and $\|\mu\|=N$. We may choose $\mu$ to satisfy the additional condition $\mu(\{0\})=0$. Indeed, suppose $\mu(\{0\})=c \neq 0$. Then 0 is not a peak point for $A(\bar{D})$, so there exists a probability measure $\nu$ on $\partial D$ such that $\int g d \nu=g(0)$ for all $g \in A(\bar{D})$ and $\nu(\{0\})=0$. (A reference for these facts is [29, pp. 4-8].) Let $\mu^{\prime}=\left(\mu-c \delta_{0}\right)+c \nu$. Clearly, if $g \in A(\bar{D}), \int g d \mu=\int g d \mu^{\prime}$ and furthermore,

$$
\left\|\mu^{\prime}\right\|=\left\|\left(\mu-c \delta_{0}\right)+c \nu\right\| \leqq\left\|\mu-c \delta_{0}\right\|+c=(\|\mu\|-c)+c=\|\mu\| ;
$$


finally, $\mu^{\prime}(\{0\})=0$. Since $d \mu^{\prime}-f d s$ annihilates $A(\bar{D})$, it follows from 6.3 below that $d \mu^{\prime}-f d s=k d s, k \in L^{1}(\partial D ; d s)$. Moreover, $\int h k d s=0$ for every $h \in B(D)$, by 3.1 . Hence, $\int h f d s=\int h d \mu^{\prime}$ for each $h \in B(D)$, so that

$$
\sup \left\{\left|\int h f d s\right|: h \in B(D),\|h\|_{\infty} \leqq 1\right\} \leqq\left\|\mu^{\prime}\right\|=N
$$

as required.

COROLlaRY 3.7. $B(D)$ is the weak ${ }^{*}$ closure in $L^{\infty}(\partial D ; d s)$ of the set of rational functions with poles off $\bar{D}$. The functions $f_{n}$ in 3.6 may be chosen to be rational functions.

Proof. By 2.4, rational functions analytic on $\bar{D}$ are uniformly dense in $A(\bar{D})$.

4. The maximal ideal space. If $R$ is a bounded domain and $z \in R$ then evaluation at $z$ gives rise to a complex homomorphism $\phi_{z}$ of $B(R)$ defined by $\phi_{z}(f)=f(z)$. Thus, $R$ can be regarded as a subset of $M=M(R)$, the maximal ideal space of $B(R)$. In fact, the map $z \rightarrow \phi_{z}$ embeds $R$ homeomorphically as an open subset $\hat{R}$ of $M$ $[17$, p. 160]. It is natural to ask if the closure of $\hat{R}$ (in $M$ ) is all of $M$. Even in the simplest of cases, this question, the so-called corona conjecture, is exceedingly difficult. For $R=\Delta$, the open unit disc, the answer is affirmative [5]. Armed with this fact, it is rather easy to show that the answer remains yes if $R$ is a finite open Riemann surface (in particular, a plane domain with finite connectivity). On the other hand, virtually nothing seems to be known about the case of infinite connectivity: at present there are no examples of planar sets of infinite connectivity for which the corona conjecture is known either to be true or false. Although the results below do not shed much light on the answer to this question, they do indicate where some of the major difficulties lie. Much of our discussion will be framed in terms of a fixed domain $D$ of type $(L)$.

Recall that for $\alpha \in \bar{D}, M_{\alpha}$ denotes the set of all homomorphisms $\phi \in M$ such that $\phi(z)=\alpha$. We call $M_{\alpha}$ the fiber over $\alpha$. Since $(z-\alpha)^{-1} \in B(D)$ for $\alpha \notin \bar{D}$, it is clear that $\left\{M_{\alpha}: \alpha \in \bar{D}\right\}$ is a partition of $M$; moreover, if $\alpha \in D$, then $M_{\alpha}=\left\{\phi_{\alpha}\right\}$, as we have observed above.

If $\zeta \in \partial D \backslash\{0\}$, it is easy to see that $M_{\zeta}$ is naturally homeomorphic to the fiber over 1 in the maximal ideal space of $H^{\infty}$. This follows, for instance, from the fact that each $f \in B(D)$ has the representation $f=f_{n}+f_{n}$, where $f_{n}$ is analytic on a neighborhood of $\gamma_{n}$ while $f_{n}$ is bounded and analytic on the (simply-connected) component of the complement of $\gamma_{n}$ in the sphere which intersects $D$. Explicitly,

$$
f_{n}(z)=\frac{1}{2 \pi i} \int_{\gamma_{n}} \frac{f(\zeta)}{\zeta-z} d \zeta, \quad f_{n}(z)=\frac{1}{2 \pi i} \int_{\Gamma \mid \gamma_{n}} \frac{f(\zeta)}{\zeta-z} d \zeta .
$$

The reasoning for the disc also shows that, for each $n, \bigcup_{\zeta \in \gamma_{n}} M_{\zeta}$ is connected and homeomorphic to $M(\Delta) \backslash \Delta$. Furthermore, the Shilov boundary $X$ of $B(D)$ is the maximal ideal space of $L^{\infty}(\partial D ; d s)$. These facts and their unstated ramifications 
follow in a routine fashion from the $H^{\infty}$ theory, so we shall omit their proofs. What should be obvious at this point is that for domains of type $(L)$ the only point where anything new can happen is the origin. Accordingly, we shall concentrate our attention at that point.

The first observation worth making is

Proposition 4.1. $\hat{D}$ is dense in $M \backslash M_{0}$.

Proof. Let $\phi \in M_{\zeta}, \zeta \in \partial D \backslash\{0\}$; we claim there exists a net $\left\{z_{i}\right\} \subset D$ such that $\phi_{z_{i}} \rightarrow \phi$. To simplify notation we may assume that $\zeta \in \gamma_{0}$. Let $\psi$ denote the restriction of $\phi$ to $H^{\infty}=B(\Delta)$. By the corona theorem there exists a net $\left\{z_{i}\right\}$ in $\Delta$ such that $\phi_{z_{i}} \rightarrow \psi$ in $M(\Delta)$. In particular, $\phi_{z_{i}}(z) \rightarrow \psi(z)=\zeta$ so that $z_{i} \rightarrow \zeta$. Thus, we may assume $\left\{z_{i}\right\} \subset D$. Let $f \in B(D)$. We have

and

$$
\phi(f)=\phi\left(f_{0}+\tilde{f}_{0}\right)=\phi\left(f_{0}\right)+\phi\left(\tilde{f}_{0}\right)=\psi\left(f_{0}\right)+\tilde{f}_{0}(\zeta)
$$

$$
\phi_{z_{i}}(f)=f_{0}\left(z_{i}\right)+\tilde{f}_{0}\left(z_{i}\right) \rightarrow \psi\left(f_{0}\right)+\tilde{f}_{0}(\zeta),
$$

so that $\phi_{z_{i}}(f) \rightarrow \phi(f)$ for each $f \in B(D)$, as required.

The argument above, dependent as it is on the case of the disc, disguises the essential difficulty involved in the corona conjecture. The problem in question is made more explicit in

Proposition 4.2. These are equivalent:

(1) $\hat{R}$ is dense in $M(R)$.

(2) Given $f_{1}, f_{2}, \ldots, f_{n} \in B(R)$ such that

$$
\left|f_{1}(z)\right|+\left|f_{2}(z)\right|+\cdots+\left|f_{n}(z)\right| \geqq \delta>0, \quad z \in R,
$$

there exist $g_{1}, g_{2}, \ldots, g_{n} \in B(R)$ such that

$$
f_{1} g_{1}+f_{2} g_{2}+\cdots+f_{n} g_{n} \equiv 1 \text { on } R \text {. }
$$

Proof. This is an easy consequence of the definition of the topology of $M(R)$. For details, see [17, p. 163].

Even very weak versions of condition (2) of 4.2 yield interesting information about $B(R)$. For instance, we have

Proposition 4.3. Let $R$ be a bounded plane domain, $\zeta \in \partial R$. Then the following conditions are equivalent.

(1) If $f \in B(R)$ satisfies $|z-\zeta|+|f(z)| \geqq \delta>0$ on $R$ then there exist $g, h \in B(R)$ such that $(z-\zeta) g+f h \equiv 1$ on $R$.

(2) If $f \in B(R)$ then $\hat{f}\left(M_{\zeta}\right) \subset \mathrm{Cl}(f ; \zeta)$.

Proof. Suppose (2) is false. Then there exists $f \in B(R)$ such that $0 \in \hat{f}\left(M_{\zeta}\right)$ but $0 \notin \mathrm{Cl}(f ; \zeta)$. Clearly, $f$ satisfies the condition of (1) for an appropriately small $\delta$, so $(z-\zeta) g+f h \equiv 1$ for some $g, h \in B(R)$. Choosing $\phi \in M_{\zeta}$ such that $\phi(f)=\hat{f}(\phi)=0$, we obtain a contradiction. Conversely, if (1) is false for some $f \in B(R)$ it follows 
from the Gelfand-Mazur theorem that there exists $\phi \in M$ such that $\phi(f)=\phi(z-\zeta)$ $=0$. Clearly $\phi \in M_{\zeta}$. It is obvious that $0 \in \hat{f}\left(M_{\zeta}\right) \mid \mathrm{Cl}(f ; \zeta)$.

It would be extremely interesting to have an example of a domain for which conditions (1) and (2) above do not hold. For such a domain, of course, the corona conjecture would fail in the worst possible way. We should point out that the inclusion $\mathrm{Cl}(f ; \zeta) \subset \hat{f}\left(M_{\zeta}\right)$ always holds. Indeed, suppose $z_{n} \rightarrow \zeta$ and $f\left(z_{n}\right) \rightarrow \beta$. Let $I$ be the ideal in $B(R)$ of all functions $h$ such that $h\left(z_{n}\right) \rightarrow 0$. I is a proper ideal and is thus contained in a maximal ideal, i.e., there exists $\phi \in M(R)$ such that $\phi(h)=0$ for all $h \in I$. Since $z-\zeta, f-\beta \in I$, we have $\phi(z-\zeta)=0, \phi(f-\beta)=0$. The first equality shows that $\phi \in M_{\zeta}$ and the second gives $\phi(f)=\beta$.

When $R=\Delta$, it is known that (1) of 4.3 is true. Of course, this follows from the corona theorem, but it can also be obtained as a consequence of the RieszNevanlinna factorization theory for functions in $H^{\infty}$ (see [17, p. 162] for details). Hence, if $D$ is a domain of type $(L)$ and $\zeta \in \partial D \mid\{0\}$ it follows (by decomposing integrals, for instance) that $\hat{f}\left(M_{\zeta}\right)=\mathrm{Cl}(f ; \zeta)$. The proof breaks down for $\zeta=0$; entirely different techniques are required to treat this point.

Proposition 4.4. Let $f \in B(D)$ and suppose $|z|+|f(z)| \geqq \delta>0$ for $z \in D$. Then there exist $g, h \in B(D)$ such that $f(z) g(z)+z h(z) \equiv 1, z \in D$.

Proof. Let $\gamma=\{|z|=\varepsilon\}$ be a circle in $D$ such that $|f(z)|>\delta / 2$ on and inside $\gamma$. Let $\Gamma_{\gamma}$ be that part of $\partial D$ lying inside of $\gamma$ and set

$$
g(z)=\frac{1}{2 \pi i} \int_{\gamma} \frac{1}{f(\zeta)} \frac{d \zeta}{\zeta}-\frac{1}{2 \pi i} \int_{\Gamma_{y}} \frac{1}{f(\zeta)} \frac{d \zeta}{\zeta-z}
$$

where the integrals are taken in the counterclockwise direction. Then

$$
g(z)-\frac{1}{f(z)}=-\frac{1}{2 \pi i} \int_{\gamma} \frac{z}{f(\zeta)} \frac{d \zeta}{\zeta(\zeta-z)}=O(|z|)
$$

as $z \rightarrow 0$. It follows from (1) that $g$ is analytic off the compact set bounded by $\Gamma_{\gamma}$, and it is clear from (2) and the maximum modulus principle that $g$ is bounded; hence $g \in B(D)$. Since $f$ is bounded away from 0 near 0 we have $g(z) f(z)-1$ $=O(|z|), z \rightarrow 0$. Set $h(z)=(1-g(z) f(z)) / z$. Done.

The elegant proof of 4.4 was communicated to me by Lennart Carleson.

Combining 4.3 and 4.4 we obtain

Proposition 4.5. Let $f \in B(D)$. Then $\hat{f}\left(M_{0}\right)=\mathrm{Cl}(f ; 0)$.

Corollary 4.6. $M_{0}$ is connected.

Proof. (Cf. [18, p. 188].) First of all, we note that $M_{0}$ is a hull; indeed, it is the zero set of the function $\hat{z}$. Hence [20, p. 116], $M_{0}$ is the maximal ideal space of the Banach algebra $B(D) / I$, where $I$ is the ideal of all functions whose Gelfand transforms vanish identically on $M_{0}$. Suppose $M_{0}=K_{0} \cup K_{1}$, where the $K_{j}$ are disjoint compact sets. By the Shilov idempotent theorem [20, p. 168], there exists $\tilde{f} \in B(D) / I$ 
such that $\tilde{f}^{\wedge}=j$ on $K_{j}$. Let $\pi: B(D) \rightarrow B(D) / I$ be the quotient map, and choose $f \in B(D)$ such that $\pi(f)=f$. Clearly, $\hat{f}\left(M_{0}\right)=\{0,1\}$. But $\hat{f}\left(M_{0}\right)=\mathrm{Cl}(f ; 0)$ by 6.5 , and $\mathrm{Cl}(f ; 0)$ is connected since $D$ is locally connected at $0[5$, p. 3]. This contradiction shows that $M_{0}$ must be connected.

There is an alternative argument, independent of Proposition 4.4, which is also worth presenting. We argue as above to conclude that $\hat{f}\left(M_{0}\right)=\{0,1\}$. Since $\mathrm{Cl}(f ; 0)$ is connected and contained in $\hat{f}\left(M_{0}\right)$ it must be a singleton, say $\mathrm{Cl}(f ; 0)=\{0\}$. We will show that $K_{1}$ does not meet the closure cl $(D)$ of $D$ in $M$. First of all, note that since $K_{1}=M_{0} \cap\{\hat{f}=1\}$, we must have $\hat{f}(\phi)=1$ for each $\phi \in K_{1}$. Suppose that $\phi \in K_{1} \cap \operatorname{cl}(D)$. Then there exists a net $\left\{z_{i}\right\}$ in $D$ such that $\phi_{z_{i}} \rightarrow \phi$ in $M$; i.e., $h\left(z_{i}\right) \rightarrow \hat{h}(\phi)$ for each $h \in B(D)$. Taking $h=z$, we have $z_{i} \rightarrow 0$ in $D$. Thus, $f\left(z_{i}\right) \rightarrow 0$ since 0 is the unique cluster value of $f$ at 0 . This contradicts $f\left(z_{i}\right) \rightarrow \hat{f}(\phi)=1$. Thus $K_{1} \cap \operatorname{cl}(D)=\varnothing$. We now obtain a contradiction by observing that the Shilov boundary of $B(D)$ is contained in $\mathrm{cl}(D)$, yet meets each component of $M$.

The content of the preceding paragraph can be summarized in

Proposition 4.7. Let $R$ be a bounded plane domain, $f \in B(R)$. If $\zeta \in \partial R$ and $R$ is locally connected at $\zeta$ then $M_{\zeta}$ is connected.

It is easy to see that if $R$ fails to be locally connected at $\zeta, M_{\zeta}$ can be disconnected. In fact, the example of 2.3 shows that this may happen even if $R$ is the interior of its closure.

In concluding this section, let us point out that when the conditions of 4.3 are satisfied the Gelfand theory allows us to reduce (2) of 4.2 to a local condition. More precisely, we have

Proposition 4.8. Let $R$ be a bounded plane domain and suppose $\hat{f}\left(M_{\zeta}\right)=\mathrm{Cl}(f ; \zeta)$ for every $f \in B(R)$ and every $\zeta \in \partial R$. Then these are equivalent:

(1) $\hat{R}$ is dense in $M(R)$.

(2) Given $f_{1}, f_{2}, \ldots, f_{n} \in B(R)$ such that

$$
\left|f_{1}(z)\right|+\left|f_{2}(z)\right|+\cdots+\left|f_{n}(z)\right| \geqq \delta>0, \quad z \in R,
$$

there exist for each $\zeta \in \partial R$ functions $g_{1}(z ; \zeta), g_{2}(z ; \zeta), \ldots, g_{n}(z ; \zeta) \in B(R)$ such that

$$
h_{\zeta}(z)=f_{1}(z) g_{1}(z ; \zeta)+\cdots+f_{n}(z) g_{n}(z ; \zeta)
$$

is bounded away from 0 in a neighborhood (in $R$ ) of $\zeta$.

Proof. Clearly, the $f_{j}$ do not all vanish at any point of $R$. If $\phi \in M_{\zeta}(\zeta \in \partial R)$, then $\phi\left(f_{j}\right)=0,1 \leqq j \leqq n$, implies that $\phi\left(h_{\zeta}\right)=0$; but $\phi\left(h_{\zeta}\right) \neq 0$, since $0 \notin \mathrm{Cl}\left(h_{\zeta} ; \zeta\right)$. Hence, if (2) holds the $f_{j}$ belong to no common maximal ideal. It follows that there exist $g_{1}, g_{2}, \ldots, g_{n} \in B(R)$ such that $f_{1} g_{1}+f_{2} g_{2}+\cdots+f_{n} g_{n} \equiv 1$. By 4.2, we are done.

In particular, it follows from 4.8 and 4.4 that for domains of type $(L)$ it is enough to exhibit functions $g_{1}, \ldots, g_{n} \in B(D)$ such that

$$
\left|f_{1}(z) g_{1}(z)+\cdots+f_{n}(z) g_{n}(z)\right| \geqq \eta>0 \text { near } 0
$$

for some $\eta>0$ (the $f_{j}$ 's having been given). 
5. A distinguished homomorphism. In this section we shall be concerned with the problem of how the metric structure of a domain affects its function theory. Throughout, $D$ will be a domain of type $(L)$ with boundary curves $\gamma_{0}=\{|z|=1\}$, $\gamma_{n}=\left\{\left|z-x_{n}\right|=r_{n}\right\}, n=1,2, \ldots$

Let $Y$ be a compact set in the plane, $x \in Y$. We say $x$ is a peak point for $A(Y)$ if there exists a function $f \in A(Y)$ such that (1) $f(x)=1$ and (2) $|f(z)|<1$ for $z \in Y \backslash\{x\}$. If $R$ is a domain bounded by finitely many simple closed curves it is easy to see that each point of $\partial R$ is a peak point for $A(\bar{R})$. For domains of infinite connectivity, however, not every point of the boundary need be a peak point. In fact, we have

THEOREM 5.1. 0 is a peak point for $A(\bar{D})$ if and only if

$$
\sum_{n=1}^{\infty} \frac{r_{n}}{x_{n}}=\infty
$$

Proof. The proof is immediate from [29, p. 58] and 2.4.

Theorem 5.1 should be compared with Beck's theorem [3] on "peak points" for bounded analytic functions. We should also point out that nothing so simple as 5.1 is true even for domains whose structure is very close to that of domains of type $(L)$; for details see [29, pp. 60-62].

Now suppose that $D$ is given and 0 is not a peak point for $A(\bar{D})$. Then $\sum r_{n} / x_{n}<\infty$, so $d \mu(\zeta)=(2 \pi i)^{-1} \zeta^{-1} d \zeta$ is a finite measure on $\partial D$. By Cauchy's theorem, $r(0)=$ $\int_{\Gamma} r(\zeta) d \mu(\zeta)$ for each rational function analytic on $\bar{D}$; hence, by Theorem 2.4 we have

$$
f(0)=\int_{\Gamma} f(\zeta) d \mu(\zeta), \quad f \in A(\bar{D})
$$

It follows that the measure $\mu$ is multiplicative on $A(\bar{D})$. This property persists in the (weak *) limit, so that, by 3.1 ,

$$
\int_{\Gamma} g h d \mu=\left(\int_{\Gamma} g d \mu\right)\left(\int_{\Gamma} h d \mu\right), \quad g, h \in B(D) .
$$

Thus, $\phi_{0}(h)=\int h d \mu$ is a complex homomorphism of $B(D)$; and, since $\phi_{0}(z)=0$ $\phi_{0} \in M_{0}$. If $K d s$ is another measure which represents evaluation at 0 on $A(\bar{D})$

$$
f(0)=\int_{\Gamma} f(\zeta) K(\zeta) d s, \quad f \in A(\bar{D}),
$$

the same argument shows that $\psi_{K}$, given by

$$
\psi_{K}(h)=\int_{\Gamma} h(\zeta) K(\zeta) d s, \quad h \in B(D),
$$

is an element of $M_{0}$. In fact, $\phi_{0}=\psi_{K}$ for any such $K$. Indeed, writing

$$
(2 \pi i)^{-1} \zeta^{-1} d \zeta-K d s=H d s, \quad H \in L^{1}(d s),
$$


we have

$$
\int_{\Gamma} f(\zeta) H(\zeta) d s=0, \quad f \in A(\bar{D}) .
$$

Since $A(\bar{D})$ is weak * dense in $B(D)$

$$
\int_{\Gamma} h(\zeta) H(\zeta) d s=0, \quad h \in B(D)
$$

which proves our assertion.

Thus, if $\sum r_{n} / x_{n}<\infty$, there exists a unique complex homomorphism $\phi_{0}$ in $M_{0}$ which arises as the extension to $B(D)$ of those complex measures which represent evaluation at 0 on $A(\bar{D})$ and are absolutely continuous with respect to arc length. The remainder of this section will be devoted to the study of this distinguished homomorphism. We have

THEOREM 5.2. The point $0 \in \partial D$ fails to be a peak point for $A(\bar{D})$ if and only if $\lim _{x \rightarrow 0-} h(x)$ exists for every $h \in B(D)$. In this case, the limit actually exists for approach off any wedge $|\theta|<\delta$, and one has

$$
\phi_{0}(h)=\lim _{x \rightarrow 0-} h(x) .
$$

Proof. First suppose 0 is a peak point for $A(\bar{D})$ and let $f$ be a function that peaks there. Let the sequence $\left\{z_{n}\right\} \subset D, z_{n} \rightarrow 0$ be given. Clearly, we can choose a subsequence $\left\{z_{n_{k}}\right\}$ such that $f\left(z_{n_{k}}\right) \rightarrow 1$ as rapidly as we like; in particular, the $z_{n_{k}}$ may be chosen in such a way that $\left\{f\left(z_{n_{k}}\right)\right\}$ is an interpolating sequence for $H^{\infty}$ [17, pp. 194-206]. Let $h \in H^{\infty}$ be such that $h\left(f\left(z_{n_{k}}\right)\right)=(-1)^{k}$. Then $h \circ f \in B(D)$ and $h \circ f$ does not tend to a limit on $\left\{z_{n}\right\}$. Thus, for any curve in $D$ with an endpoint at 0 there exists a function in $B(D)$ which fails to tend to a limit along that curve.

Suppose next that 0 is not a peak point for $A(\bar{D})$. Then $\sum r_{n} / x_{n}<\infty$, and so $\sum r_{n} /\left(x_{n}-r_{n}\right)<\infty$ (since $\left.r_{n}<x_{n}\right)$. Let

$$
f_{n}(z)=\frac{1}{2 \pi i} \int_{\gamma_{n}} \frac{f(\zeta)}{\zeta-z} d \zeta, \quad f \in B(D)
$$

Then by Cauchy's formula $f(z)=\sum_{n=0}^{\infty} f_{n}(z)$ for $z \in D$. Also,

$$
\phi_{0}(f)=\frac{1}{2 \pi i} \int_{\Gamma} \frac{f(\zeta)}{\zeta} d \zeta=\sum_{n=0}^{\infty} f_{n}(0) .
$$

For $-\frac{1}{2} \leqq x \leqq 0$, we have

$$
\left|f_{n}(x)\right| \leqq(2 \pi)^{-1}\|f\|_{\infty} 2 \pi r_{n}\left(x_{n}-r_{n}\right)^{-1}, \quad n=1,2, \ldots,
$$

independently of $x$. Thus

$$
\sum_{n=N}^{\infty}\left|f_{n}(x)\right| \leqq\|f\|_{\infty} \sum_{n=N}^{\infty} \frac{r_{n}}{x_{n}-r_{n}} \rightarrow 0
$$


as $N \rightarrow \infty$, uniformly on $\left[-\frac{1}{2}, 0\right]$. Therefore,

$$
\lim _{x \rightarrow 0-} f(x)=\lim _{x \rightarrow 0-} \sum_{n=0}^{\infty} f_{n}(x)=\sum_{n=0}^{\infty} f_{n}(0)=\phi_{0}(f),
$$

as required. It now follows by a classical theorem of Lindelöf (cf. [26, p. 306]) that the limit actually exists off any wedge containing the positive real axis. Alternatively, one can show directly that if $\delta>0$ is given there exists $\varepsilon>0$ such that $\sum_{n=0}^{\infty} f_{n}(z)$ converges uniformly on the set $\{z:|z| \leqq \varepsilon$ and $|\arg z| \geqq \delta\}$. The result then follows as above.

Henceforth, we shall assume that 0 is not a peak point for $A(D)$, so that the complex homomorphism $\phi_{0}$ exists.

At this point, it should be clear that, in some sense, $\phi_{0}$ is related to the homomorphisms $\phi_{z}, z \in D$. Part of our problem is to make this statement precise. Of course, Theorem 5.2 allows us to conclude that $\phi_{0}$ lies in the sequential closure of $\hat{D}$; however, much more is true. It turns out that the relevant concept is the familiar notion of (Gleason) part. Recall that two complex homomorphisms, $\phi$ and $\psi$, of a function algebra $\mathfrak{A}$ lie in the same part $(\phi \sim \psi)$ if and only if $\|\phi-\psi\|<2$. Equivalently, $\phi \sim \psi$ if and only if

$$
\sup \{|\psi(f)|: f \in \mathfrak{A},\|f\| \leqq 1, \phi(f)=0\}<1 .
$$

$\sim$ is an equivalence relation, and the parts of $\mathfrak{A}$ are the equivalence classes into which it partitions the maximal ideal space of $\mathfrak{A}$. If $\mathfrak{A}=B(D)$, it is easy to see that all of $D$ (i.e., $\hat{D}$ ) lies in a single part; this follows, for instance, from Schwarz's lemma applied to a chain of discs. Our claim is

THEOREM 5.3. The homomorphism $\phi_{0}$ lies in the same Gleason part as D.

For the proof, we shall need the following result, itself of some interest.

Proposition 5.4. Let $\left\{f_{n}\right\} \subset B(D)$ and suppose $f_{n}(z) \rightarrow f(z), z \in D$, pointwise boundedly. Then $\phi_{0}\left(f_{n}\right) \rightarrow \phi_{0}(f)$.

Proof. Obviously, we may assume $\left\|f_{n}\right\|_{\infty} \leqq 1$. By Vitali's theorem, $f_{n}(z) \rightarrow f(z)$ uniformly on compact subsets of $D$. Choose disjoint curves $\gamma_{n}^{\prime}=\left\{\left|z-x_{n}\right|=r_{n}^{\prime}\right\}$ $(n=1,2, \ldots)$ in $D$ so that $\sum r_{n}^{\prime} / x_{n}<\infty$ and $\bigcup_{k \neq n} \gamma_{k}^{\prime}$ lies in the unbounded component of the complement of $\gamma_{n}^{\prime}$; let $\gamma_{0}^{\prime} \subset D$ be a circle centered at 0 which surrounds $\bigcup_{n=1}^{\infty} \gamma_{n}^{\prime}$. We write $\Gamma_{N}^{\prime}=\bigcup_{n=0}^{N} \gamma_{n}^{\prime}, \Gamma^{\prime}=\{0\} \cup \bigcup_{n=0}^{\infty} \gamma_{n}^{\prime}$. Let $D^{\prime}$ be the subdomain of $D$ which has $\Gamma^{\prime}$ as its boundary. Then for $z \in D^{\prime}$ and $g \in B(D)$ we have

$$
g(z)=\frac{1}{2 \pi i} \int_{\Gamma^{\prime}} \frac{g(w)}{w-z} d w
$$

Let $\varepsilon>0$ be given and choose $N$ so large that

$$
\frac{1}{2 \pi} \int_{\Gamma^{\prime} \mid \Gamma^{\prime}} \frac{d s}{|w|}<\varepsilon
$$


This is possible since $\sum r_{n}^{\prime} / x_{n}<\infty$. Now if $f \in B(D)$ it is clear that

Hence,

$$
\phi_{0}(f)=\frac{1}{2 \pi i} \int_{\Gamma} \frac{f(w)}{w} d w .
$$

$$
\left|\phi_{0}(f)-\phi_{0}\left(f_{k}\right)\right| \leqq\left|\frac{1}{2 \pi i} \int_{\Gamma_{N}^{\prime}} \frac{f(w)-f_{k}(w)}{w} d w\right|+2 \varepsilon .
$$

Since $\Gamma_{N}^{\prime}$ is a compact subset of $D, f_{k}(w) \rightarrow f(w)$ uniformly on $\Gamma_{N}^{\prime}$ as $k \rightarrow \infty$; thus

$$
\limsup _{k \rightarrow \infty}\left|\phi_{0}(f)-\phi_{0}\left(f_{k}\right)\right| \leqq 2 \varepsilon .
$$

But $\varepsilon$ was arbitrary, so $\phi_{0}(f)=\lim \phi_{0}\left(f_{k}\right)$, as required.

Actually, even more is true: $\phi_{0}$ remains continuous when $B(D)$ is endowed with the strict topology ( $\beta$-topology) [21]. This observation has also been made (independently) by Rudin [24].

Proof of 5.3. Fix $x \in D$. We must show

$$
\sup \left\{|f(x)|: f \in B(D),\|f\|_{\infty} \leqq 1, \phi_{0}(f)=0\right\}<1 .
$$

Suppose, to the contrary, that there exists a sequence of functions $\left\{f_{n}\right\}$ in $B(D)$ such that $\phi_{0}\left(f_{n}\right)=0,\left\|f_{n}\right\| \leqq 1$, and $f_{n}(x) \rightarrow 1$ as $n \rightarrow \infty$. $\left\{f_{n}\right\}$ is a normal family, so by extracting a subsequence and renumbering we have $f_{n}(z) \rightarrow f(z)$ uniformly on compact subsets of $D$. Of course, $f \in B(D)$ and $\|f\|_{\infty} \leqq 1$; since $f(x)=1$, it follows that $f(z) \equiv 1$. By 5.4

$$
0=\lim \phi_{0}\left(f_{n}\right)=\phi_{0}(f)=\phi_{0}(1)=1,
$$

a contradiction.

Corollary 5.5. Evaluation at 0 lies in the same part of $A(\bar{D})$ as $D$.

The identification of the parts of $B(D)$ remains an open question. Since each point of $\partial D \mid\{0\}$ is a peak point for $A(\bar{D})$, any part which lies in $\bigcup\left\{M_{\zeta}: \zeta \in \partial D \mid\{0\}\right\}$ must actually lie in a single fiber. In this case, the problem reduces to the identification of the parts of $H^{\infty}$, for which see Hoffman [18]. Thus, only those parts which contain points of $M_{0}$ need be considered.

To conclude this section, we prove

THEOREM 5.6. $\phi_{0}$ does not lie in the Shilov boundary of $B(D)$.

Proof. By [10], there exists $f \in B(D)$ such that $f$ is nonconstant and continuous on $\bar{D} \mid\{0\}$ and $|f|=1$ on $\partial D \mid\{0\}$. Since the Shilov boundary $X$ of $B(D)$ is just the maximal ideal space of $L^{\infty}(\partial D ; d s)(\S 4),|\hat{f}(\psi)|=1$ for any $\psi \in X$. On the other hand, since $\phi_{0}$ lies in the same part as $D,\left|\phi_{0}(f)\right|<1$. Hence $\phi_{0} \notin X$.

A similar proof, not depending on the results of [10], can be based on an abstract extremal technique due to Bishop [4]. 
It is worth noting that for $f$ as above $\mathrm{Cl}(f ; 0)=\{|z| \leqq 1\}$. Indeed, it is clear that $\mathrm{Cl}_{\mathrm{B}}(f ; 0) \subset\{|z|=1\}$; and by $2.5 \partial(\mathrm{Cl}(f ; 0)) \subset \mathrm{Cl}_{\mathrm{B}}(f ; 0)$. Since $f$ is bounded and $\phi_{0}(f) \in \mathrm{Cl}(f ; 0) \mid \mathrm{Cl}_{\mathrm{B}}(f ; 0)$ it follows that $\mathrm{Cl}(f ; 0)=\{|z| \leqq 1\}$.

6. A new algebra. Several years ago, Allen Devinatz asked for a characterization of the (uniform) closure in $L^{\infty}$ of the set of bounded measurable functions on the circle only finitely many of whose negative Fourier coefficients fail to vanish (Bull. Amer. Math. Soc. 71 (1965), p. 855). Recently, Donald Sarason [25], [16] answered this question in the following way.

THEOREM 6.1. $\left[H^{\infty}, \bar{z}\right]=H^{\infty}+C$.

Here, $\left[H^{\infty}, \bar{z}\right]$ denotes the uniformly closed subalgebra of $L^{\infty}(d \theta)$ generated by $\bar{z}$ and the elements of $H^{\infty}$, and $C=C\left(\gamma_{0}\right)$ is the algebra of all continuous functions on the unit circle. The assertion of the theorem is that each element of $\left[H^{\infty}, \bar{z}\right]$ can be written as the sum of an $H^{\infty}$ function and a continuous function. This representation is, of course, far from unique since $H^{\infty} \cap C=A$ is large. Not the least remarkable feature of 6.1 is the (implicit) fact that $H^{\infty}+C$ is an algebra, a result which is by no means obvious.

We are interested in extending 6.1 and its corollary 6.2 to our more general domains. Since part of the purpose of doing this is to indicate how the infinitely connected case differs from that of the disc, it will be instructive to include a proof of 6.1. Our proof is somewhat different from Sarason's and may be of independent interest.

Proof of 6.1. By the Weierstrass theorem, $H^{\infty}+C \subset\left[H^{\infty}, \bar{z}\right]$. On the other hand, $\left[H^{\infty}, \bar{z}\right]$ obviously coincides with the uniformly closed algebra generated by $H^{\infty}$ and $C$; hence, it is enough for our purposes to show that $H^{\infty}+C$ is a closed subalgebra of $L^{\infty}$. Let $P$ be the subset of $C$ consisting of trigonometric polynomials. It is easy to see that $H^{\infty}+P$ is an algebra, and that that algebra is dense in $H^{\infty}+C$. Thus, to conclude that $H^{\infty}+C$ is a closed algebra we need only show that $H^{\infty}+C$ is uniformly closed.

We have

$$
C \stackrel{i}{\longrightarrow} L^{\infty} \stackrel{\pi}{\longrightarrow} L^{\infty} / H^{\infty} \stackrel{\pi^{-1}}{\longrightarrow} L^{\infty},
$$

where $i$ is the canonical injection and $\pi$ is the quotient map. Clearly, $\pi^{-1}(\pi(i(C)))$ $=H^{\infty}+C$; thus $H^{\infty}+C$ is closed if and only if $\pi(i(C))$ is. Let $\rho: C \rightarrow C / A$ be the natural projection. It is clear that $C / A$ and $\pi(i(C))$ are isomorphic as linear spaces under the correspondence $\Phi: \rho(f) \rightarrow \pi(i(f))$. We shall prove that $\Phi$ is a topological isomorphism; from this, it is immediate that $\pi(i(C))$ is closed, so that $H^{\infty}+C$ is closed, as required.

Let $f \in C$ and suppose $f(\zeta)=\varepsilon(\zeta)-h(\zeta), \zeta \in \gamma$, where $\varepsilon \in L^{\infty}$ and $h \in H^{\infty}$. For $k \in L^{\infty}$ denote by $k$ the harmonic extension of $k$ to $\Delta$ :

$$
\tilde{k}(z)=\frac{1}{2 \pi} \int_{0}^{2 \pi} k\left(e^{i \theta}\right) \operatorname{Re}\left\{\frac{e^{i \theta}+z}{e^{i \theta}-z}\right\} d \theta
$$


and set $k_{r}(\zeta)=\tilde{k}(r \zeta)$. Since $f$ is continuous, $f_{r} \rightarrow f$ uniformly on $\gamma_{0}$ as $r \rightarrow 1$, i.e., $(\varepsilon-h)_{r}=\left(\varepsilon_{r}-h_{r}\right) \rightarrow(\varepsilon-h)$ uniformly as $r \rightarrow 1$. Thus

$$
\left\|h_{r}-h\right\|_{\infty} \leqq\left\|\varepsilon_{r}-\varepsilon\right\|_{\infty}+\delta(r) \leqq 3\|\varepsilon\|_{\infty},
$$

where $\delta(r) \rightarrow 0$ as $r \rightarrow 1$ and $r$ is chosen so large that $\delta(r) \leqq\|\varepsilon\|_{\infty}$. Since $h_{r} \in A$, we have

$$
\inf _{g \in A}\|f+g\| \leqq\left\|(\varepsilon-h)+h_{r}\right\|_{\infty} \leqq\|\varepsilon\|_{\infty}+\left\|h-h_{r}\right\|_{\infty} \leqq 4\|\varepsilon\|_{\infty} .
$$

Since the left-hand side of $(1)$ is the norm of the coset $\rho(f)$ in $C / A$, it follows that $\left\|\pi\left(i\left(f_{n}\right)\right)\right\| \rightarrow 0$ implies $\left\|\rho\left(f_{n}\right)\right\| \rightarrow 0$. Since the reverse implication is trivial, $\Phi$ is a topological isomorphism, and we are done.

It is easy to see that $H^{\infty}+C$ has as its maximal ideal space $M(\Delta) \mid \Delta$ and that its Shilov boundary is the maximal ideal space $W$ of $L^{\infty}(d \theta)$ (cf. [17, p. 193]). Thus, we can regard the algebra of Gelfand transforms $\left(H^{\infty}+C\right)^{\wedge}$ as a closed subalgebra of $C(W)$. Let $W_{\alpha}(|\alpha|=1)$ be the fiber of $W$ over $\alpha$, i.e., the set of all complex homomorphisms $\phi \in W$ such that $\phi(z)=\alpha$. Then we have

Proposition 6.2. The algebra $\left(H^{\infty}+C\right)^{\wedge}$ consists of all functions in $C(W)$ such that for each $\alpha \in \gamma_{0}$ there exists $g_{\alpha} \in H^{\infty}$ satisfying $\hat{g}_{\alpha}=$ f on $W_{\alpha}$.

Proof. This follows from Theorem 1.1 of [14] and the observation that the fibers $W_{\alpha}$ are unions of maximal sets of antisymmetry for $\left(H^{\infty}+C\right)^{\wedge}$.

It is worth noting that 6.2 gives an "abstract" answer to the question posed by Devinatz which is independent of 6.1. For the proof, we need only replace $\left(H^{\infty}+C\right)^{\wedge}$ (wherever it appears) by $\left[H^{\infty}, \bar{z}\right]^{\wedge}$.

Now suppose $R$ is a finitely connected region whose boundary $\partial R$ consists of finitely many disjoint simple closed curves. It is easy to extend 6.1 (and 6.2) to this case. For simplicity, we may formulate our assertion as $[B(R), C(\partial R)]=$ $B(R)+C(\partial R)$. Here all functions are understood to be defined on $\partial R$, and $[B(R), C(\partial R)]$ is the smallest closed subalgebra of $L^{\infty}(\partial R ; d s)$ which contains both $B(R)$ and $C(\partial R)$. However, we shall not insist on the details of the proof since another proof of this result will appear (implicitly) below.

When $R$ is a domain of infinite connectivity the techniques that make possible the reduction of the finitely connected case to the situation on the disc are no longer available, and one must proceed differently. Below, we shall prove the analogue of 6.1 for domains $D$ of type $(L)$. In the process, we shall obtain a proof for the finitely connected case that differs somewhat from that already given. We shall continue, with the obvious modifications, the notation established above.

First of all, we need the following generalization of the classical F. and M. Riesz theorem [17, p. 46].

Proposition 6.3. Let $\mu$ be a finite complex Baire measure on $\partial D$ and suppose $\int_{\Gamma} f(\zeta) d \mu(\zeta)=0$ for each $f \in A(\bar{D})$. Then $d \mu=k d s+c \delta_{0}$, where $k \in L^{1}(d s)$, c is a complex constant, and $\delta_{0}$ is the point mass at 0. 
Proof. Fix $n$, and let $\mu_{n}$ be the restriction of $\mu$ to $\Gamma_{n}, \tilde{\mu}_{n}=\mu-\mu_{n}$. By the balayage formula (5) of 3.2 we have

$$
\int g(\xi) d \tilde{\mu}_{n}(\xi)=\int_{\Gamma_{n}} g(\xi)\left(\frac{1}{2 \pi i} \int \frac{d \tilde{\mu}_{n}(\xi)}{\zeta-\xi}\right) d \zeta
$$

for each $g \in A\left(\bar{D}_{n}\right)$. Let

$$
F_{n}(\zeta)=\frac{1}{2 \pi i} \int \frac{d \tilde{\mu}_{n}(\xi)}{\zeta-\xi}
$$

Then

$$
\begin{aligned}
0 & =\int_{\Gamma} g(\zeta) d \mu(\zeta)=\int g d \mu_{n}+\int g d \tilde{\mu}_{n} \\
& =\int_{\Gamma_{n}} g(\zeta)\left[d \mu_{n}(\zeta)+F_{n}(\zeta) d \zeta\right]=\int_{\Gamma_{n}} g(\zeta) d \nu_{n}(\zeta) .
\end{aligned}
$$

Here $d \nu_{n}=d \mu_{n}+F_{n} d \zeta$. Now $\nu_{n}$ is obviously a measure on $\Gamma_{n}$ which annihilates $A\left(\bar{D}_{n}\right)$; hence, by Rudin's extension of the Riesz theorem [22] to finitely connected domains, $\nu_{n}$ is absolutely continuous with respect to arc length on $\Gamma_{n}$. Since $F_{n} d \zeta \ll d s$ by definition, it follows that $d \mu_{n} \ll d s$. Let $n \rightarrow \infty$ to conclude that $d \mu \ll d s$ on each boundary curve $\gamma_{n}$. Done.

Next, we record the following elementary fact, suggested by the referee.

Lemma 6.4. Suppose $f \in B(D)$. Then

$$
\sup \left\{|z|: z \in \mathrm{Cl}(f ; \zeta), \zeta \in \gamma_{n}\right\} \leqq\left\|\left.f\right|_{\gamma_{n}}\right\|_{\infty} .
$$

Proof. This is essentially a consequence of the Poisson integral formula. Let $J$ be a proper open subarc of $\gamma_{n}$. Pick a simply connected region $U$ in $D$ whose boundary is a simple closed curve $C$ such that $J \subset C$ and $C \mid \bar{J} \subset D$. Let $h$ be a Riemann map from $\Delta$ onto $U ; h$ extends to a homeomorphism from $\bar{\Delta}$ onto $\bar{U}$. Clearly $f \circ h \in B(\Delta)=H^{\infty}$, so if $z \in U$

$$
f(z)=f \circ h\left(h^{-1}(z)\right)=\int_{|\zeta|=1} f \circ h(\zeta) P\left(\zeta, h^{-1}(z)\right) d \lambda(\zeta),
$$

where $P$ is the Poisson kernel, $\lambda$ is normalized Lebesgue measure, and $f \circ h(\zeta)$ denotes nontangential boundary values.

Because of the reflection principle, the nontangential limits of $f$ at $\zeta_{0} \in J$ are the same as those of $f \circ h$ at $h^{-1}\left(\zeta_{0}\right)$. Also, a subset of $J$ has measure 0 if and only if its preimage under $h$ has measure zero, so

$$
\left\|\left.f \circ h\right|_{h^{-1}(J)}\right\|_{\infty}=\left\|\left.f\right|_{J}\right\|_{\infty} .
$$

Now let $\zeta_{0} \in J,\left\{z_{k}\right\} \subset D, z_{k} \rightarrow \zeta_{0}$. We may assume $\left\{z_{k}\right\} \subset U$. Then $\left\{h^{-1}\left(z_{k}\right)\right\} \subset \Delta$ and $h^{-1}\left(z_{k}\right) \rightarrow h^{-1}\left(\zeta_{0}\right)$. But examination of the Poisson kernel shows that

(2) $\quad \limsup _{k \rightarrow \infty}\left|\int_{|\zeta|=1} f \circ h(\zeta) P\left(\zeta, h^{-1}\left(z_{k}\right)\right) d \lambda(\zeta)\right| \leqq\left\|\left.f \circ h\right|_{h^{-1}(J)}\right\|_{\infty}=\left\|\left.f\right|_{J}\right\|_{\infty}$. 
The conjunction of (1) and (2) yields

as required.

$$
\limsup _{k \rightarrow \infty}\left|f\left(z_{k}\right)\right| \leqq\left\|\left.f\right|_{J}\right\|_{\infty} \leqq\left\|\left.f\right|_{\gamma_{n}}\right\|_{\infty},
$$

For convenience, we shall write $B=B(D), A=A(\bar{D}), C=C(\partial D), L^{\infty}=L^{\infty}(\partial D ; d s)$, $L^{1}=L^{1}(\partial D ; d s)$. All algebras will be viewed on the boundary.

THEOREM 6.5. $[B, C]=B+C$.

Proof. It is enough to show that $B+C$ is a closed subalgebra of $L^{\infty}$. Fix $z_{0} \in D$ and denote by $Q$ the set of all rational functions holomorphic on $\bar{D} \mid\left\{z_{0}\right\}$. By the Hartogs-Rosenthal theorem, the restriction of $Q$ to $\Gamma$ is uniformly dense in $C$. Thus, $B+Q$ is dense in $B+C$. It is also easy to see that $B+Q$ is an algebra. Indeed, let $q \in Q, h \in B$. We have $q(z)=\left(z-z_{0}\right)^{-n} q_{1}(z)$, where $q_{1} \in B \cap Q$, and $h(z)=h_{1}(z)$ $+\left(z-z_{0}\right)^{n} h_{2}(z)$, where $h_{1}$ is a polynomial of degree at most $n-1$ and $h_{2} \in B$. Hence, $q h=q_{1} h_{2}+\left(z-z_{0}\right)^{-n} q_{1} h_{1} \in B+Q$, as required. It remains only to show that $B+C$ is uniformly closed.

Let $\pi: C \rightarrow C / A$ be the quotient map and $i: C / A \rightarrow(C / A)^{* *}$ the natural (isometric) injection. Since $(C / A)^{*}=A^{\perp}$, we have $(C / A)^{* *}=\left(A^{\perp}\right)^{*}$. Let $I:\left(A^{\perp}\right)^{*} \rightarrow$ $\left(A^{\perp} \cap L^{1}\right)^{*}$ be the restriction map. Then $I:(C / A)^{* *} \rightarrow L^{\infty} /\left(A^{\perp} \cap L^{1}\right)^{\perp}=L^{\infty} / B$. Here we have used the fact that $B=\left(A^{\perp} \cap L^{1}\right)^{\perp}$, i.e. $B$ is the weak * closure of $A$ in $L^{\infty}$ (3.1). Consider now the sequence

$$
C \stackrel{\pi}{\longrightarrow} C / A \stackrel{i}{\longrightarrow}(C / A)^{* *} \stackrel{I}{\longrightarrow} L^{\infty} / B \stackrel{Q^{-1}}{\longrightarrow} L^{\infty},
$$

where $Q: L^{\infty} \rightarrow L^{\infty} / B$ is the quotient map. Obviously, the image of $C$ under this sequence of maps is (canonically isomorphic to) $B+C$. It is enough, therefore, to show that $I \circ i \circ \pi(C)$ is closed. In other words, we must show that the norms on $\pi(C)$ induced by $(C / A)^{* *}$ and $L^{\infty} / B$ are equivalent.

It will be convenient to reformulate this condition in function theoretic terms. By 6.3, each element $\mu$ of $A^{\perp}$ has the form $\mu=k d s+c \delta_{0},\|\mu\|=\int|k| d s+|c|$. Set, for $f \in C$,

$$
\begin{aligned}
& \|f\|_{1}=\sup \left\{\left|\int f d \mu\right|: \mu \in A^{\perp},\|\mu\| \leqq 1\right\}=\inf _{A}\|f+g\|_{\infty} \\
& \|f\|_{2}=\sup \left\{\left|\int f d \mu\right|: \mu \in A^{\perp} \cap L^{1},\|\mu\| \leqq 1\right\}=\inf _{B}\|f+h\|_{\infty} .
\end{aligned}
$$

We claim that $\|f\|_{1}<\lambda\|f\|_{2}$ for some universal constant $\lambda>0$. Actually, it is enough for our purposes to show that, for any sequence $\left\{f_{n}\right\} \subset C,\left\|f_{n}\right\|_{2} \rightarrow 0$ implies $\left\|f_{n}\right\|_{1} \rightarrow 0$. Of course, if $A^{\perp}=A^{\perp} \cap L^{1}$, i.e., if each measure in $A^{\perp}$ is absolutely continuous, there is nothing to prove. This situation will occur when and only when 0 is a peak point for $A$. Hence, in the sequel, we may assume that 0 is not a peak point for $A$.

Now suppose $\left\{f_{n}\right\} \subset C$ and $\left\|f_{n}\right\|_{2} \rightarrow 0$. Without loss of generality we may assume $f_{n}(0)=0$ for all $n$. Since $\left\|f_{n}\right\|_{2}=\inf _{B}\left\|f_{n}+h\right\|_{\infty}$, we have $f_{n}=\varepsilon_{n}-h_{n}$, where $\varepsilon_{n} \in L^{\infty}$, 
$h_{n} \in B,\left\|\varepsilon_{n}\right\|_{\infty} \rightarrow 0$. Let $\mu=k d s+c \delta_{0}$ be in the unit ball of $A^{\perp}$ and suppose $c \neq 0$. Then

$$
\begin{aligned}
\left|\int f_{n} d \mu\right| & =\left|\int f_{n} k d s+c f_{n}(0)\right|=\left|\int f_{n} k d s\right| \\
& \leqq\left|\int \varepsilon_{n} k d s\right|+\left|\int h_{n} k d s\right| .
\end{aligned}
$$

The first term on the right-hand side is bounded by $\left\|\varepsilon_{n}\right\|_{\infty} \int|k| d s<\left\|\varepsilon_{n}\right\|_{\infty} \rightarrow 0$. To estimate the second term, write

$$
\int h_{n} k d s=\int h_{n}(k+c K) d s-c \int h_{n} K d s,
$$

where $K d s=(2 \pi i)^{-1} \zeta^{-1} d \zeta$. Now $k+c K \in A^{\perp} \cap L^{1}$, so that the first integral above vanishes by 3.1. Hence

$$
\left|\int h_{n} k d s\right| \leqq|c|\left|\int h_{n} K d s\right| \leqq\left|\phi_{0}\left(h_{n}\right)\right| .
$$

Since $f_{n}(0)=0$, it is clear that $h_{n}$ is small (on $\Gamma$ ) near 0 . It follows from Iversen's theorem $(2.5)$ and 6.4 that $\mathrm{Cl}\left(h_{n} ; 0\right)$ can be made to lie in any disc about the origin, however small, if only $n$ is chosen large enough. Since $\phi_{0}\left(h_{n}\right) \in \mathrm{Cl}\left(h_{n} ; 0\right)$ (4.5), we are done. The proof is complete.

It should be pointed out that, in case 0 is a peak point for $A$, our proof shows that

$$
\inf _{A}\|f+g\|_{\infty}=\inf _{B}\|f+h\|_{\infty}
$$

for any $f \in C$. Actually, as Professor T. W. Gamelin pointed out, $\left({ }^{*}\right)$ remains true even if 0 fails to be a peak point. For in this case, set $d m=d s+\delta_{0}$ and let $H$ be the weak * closure of $A$ in $L^{\infty}(d m)$. One can show without difficulty that

$$
\inf _{A}\|f+g\|_{D}=\inf _{H}\|f+h\|_{L^{\infty}(d m)}, \quad f \in C .
$$

Thus, it remains to prove that $\inf _{H}\|f+h\|=\inf _{B}\|f+h\|$, and this can be done by using Iversen's theorem. Details are left to the reader. It is also worth noting that $\left(^{*}\right)$ holds true for $A=A(R), B=B(R)$, where $R$ is a finitely connected domain with smooth boundary.

Finally, let us note that the obvious analogue of 6.2 trivially holds true for our algebra $[B, C]^{\wedge}$.

7. Comments; generalizations. This section is devoted to a discussion of various extensions and generalizations of the preceding results. For convenience, the comments are grouped together by section.

\$2. A quick glance at the proof shows that 2.1 is valid for any domain $R$ whose (rectifiable) boundary consists of a union of disjoint simple closed analytic curves 
plus a set of (arc length) measure 0 . Actually, the requirement of analyticity can be weakened considerably: it is enough, for instance, that the boundary be continuously differentiable. For then, as before, $f$ will have a (unique) asymptotic value $f_{\alpha}(\zeta)$ at almost every point $\zeta$ of $\partial R$; then by Lindelöf's theorem $f(z) \rightarrow f_{\alpha}(\zeta)$ as $z \rightarrow \zeta$ nontangentially from within $R$. Of course, if the boundary is merely Jordan, $f$ will still have a unique asymptotic value at a set of points on the curve that corresponds to a set of full measure on the unit circle. Quite plainly, the existence of boundary values is a local phenomenon; the global structure of $\boldsymbol{R}$ comes into play in the requirement that the set of accumulation points of the "nice" boundary curves have zero arc length.

Generalizations of 2.2, 2.3, and 2.4 have already been discussed in $\$ 2$; accordingly, we shall offer no further comment on these results.

Iversen's theorem (2.5) is one of the most beautiful and certainly one of the most important theorems of cluster set theory; it deserves to be better known. Generalizations have proceeded in various directions, most notably by replacing $\mathrm{Cl}_{\mathbf{B}}(f ; \alpha)$ by even smaller sets. For instance, the theorem remains true if a set of capacity 0 is deleted from the boundary. A survey of what is known (stated for the case of the disc) is in [28, p. 5]. Classical cluster set theory has limited its attention almost entirely to (meromorphic) functions defined on the disc; it seems high time that more general (infinitely connected) domains were studied. Part of the reason for including the results of $\$ 5$ is to show that interesting new things can happen in the case of infinite connectivity.

§3. Theorems 3.1 and 3.6 are valid for a much larger class of domains than those of type $(L)$. For instance, the linear disposition of the holes was completely irrelevant: nothing is altered if $D$ is replaced by a domain $R$ whose boundary consists of countably many simple closed analytic curves of finite total length which accumulate at a single degenerate boundary component (a point). More generally, the boundary curves of $R$ may be allowed to accumulate at a finite number of degenerate boundary components. Indeed, if $h \in B(R)$, one can use the Cauchy integral formula to write $h=\sum_{k=1}^{n} h_{k}$, where each $h_{k}$ is analytic in a domain $R_{k} \supset R$ whose boundary accumulates at a single point. We can approximate each $h_{k}$ pointwise boundedly by functions in $A\left(\bar{R}_{k}\right)$, and the sum of these functions approximates $h$. It seems likely that 3.1 and 3.6 remain true if the set of accumulation points of the boundary has Hausdorff linear measure zero or zero analytic capacity; however, we have not pursued this line of thought to any extent. In any case, most of the argument of 3.6 remains valid if the boundary is allowed to accumulate at countably many degenerate boundary components.

We should point out that there are very definite limitations to the generality of 3.1 and 3.6. For instance, the example of 2.3 shows that neither theorem can hold in this case. Indeed, if $k=\chi_{\cup \tau_{n}}$ we have $\int_{\Gamma} k g d \zeta=0, g \in A(\bar{R})$, but $\int_{\Gamma} k f d \zeta \neq 0$. Similarly, it is easy to see (by perturbing the curves $\tau_{n}$ to the interior of $R$ ) that $f$ cannot be approximated pointwise boundedly by functions in $A(\bar{R})$. 
Since this work was done, T. W. Gamelin has developed a new approach to these problems and has succeeded in extending 3.1 and 3.6 to a class of domains much larger than that considered above. His idea is to apply the constructive techniques of Vitushkin to study the possibility of pointwise bounded approximation (on $R$ ) of functions in $B(R)$ by rational functions. These methods do not require the hypothesis of rectifiability on $\partial R$; however, the nature of the boundary remains central in determining the possibility of approximation.

$\S 4$. Questions involving fibers are intimately connected with the boundary behavior of analytic functions. Thus, it is not true that the conformal equivalence of two domains implies a topological equivalence between the fibers lying over the boundaries. A simple example may clarify the situation.

Suppose $R$ is a simply connected domain and $\phi: R \rightarrow \Delta$ maps $R$ conformally onto $\Delta$. Using classical examples from the theory of prime ends, it is easy to construct an $R$ satisfying

(1) There exists an arc $\gamma \subset \partial R$ such that $\mathrm{Cl}(\phi, \zeta)=\alpha$ for any $\zeta \in \gamma$, where $\alpha$ is some fixed point of the unit circle.

(2) There exists a point $\zeta_{0} \in \partial R$ such that $\mathrm{Cl}\left(\phi, \zeta_{0}\right)=\left\{\alpha_{i}\right\}$, where $\left\{\alpha_{i}\right\}$ is a finite, countable, or even uncountable set of points on the circle.

If $f \in B(R)$, then $T f=f \circ \phi^{-1} \in H^{\infty}$, and this correspondence is an isomorphism between these two algebras. Then for any $f \in B(R)$ we have (with the obvious notation)

$$
\begin{aligned}
\hat{f}\left(\bigcup_{\zeta \in \gamma} M_{\zeta}\right) & =(T f)^{\wedge}\left(M_{\alpha}\right) \\
\hat{f}\left(M_{\zeta_{0}}\right) & =\bigcup_{i}(T f)^{\wedge}\left(M_{\alpha_{i}}\right) .
\end{aligned}
$$

We restrict the rest of our comments to 4.4. This result is obviously local: it depends only on the nature of the boundary near the point in question. To be precise, suppose $\zeta \in \partial R$ is a boundary component and one can find a sequence of (rectifiable) curves $C_{k}$ in $R$ surrounding $\zeta$ and tending to it. Assume further that the Cauchy integral formula (2.2) is valid in the subregion of $R$ cut off by $C_{k}$; this necessitates, in particular, that the portion of $\partial R$ lying inside $C_{k}$ be rectifiable. Then clearly the argument of 4.4 applies. Actually, it is not difficult to see that the rectifiability requirement can be avoided. Indeed, suppose that $R$ is a domain such that $0 \in \partial R$ is a component of $\partial R$ and there exists an exhaustion of $R$ by subdomains $R_{n}$, each of which has rectifiable boundary, such that 0 is a component of $\partial R_{n}$. For a fixed $f \in B(R)$ the argument of 4.4 gives functions $g_{n}, h_{n} \in B\left(R_{n}\right)$ such that $f g_{n}+z h_{n}=1$ on $R_{n}$, and these functions are uniformly bounded. It follows that there exist $g, h \in B(R)$ such that $f g+z h=1$ on $R$, as required. (We have assumed that the representation of 2.2 holds on a suitable subregion of each $R_{n}$.)

$\S 5$. For further information on peak points for algebras of analytic functions see [29] and the references cited there. 
A few comments on Theorem 5.2 are in order. For certain domains, the asymptotic value $\phi_{0}(f)$ may exist for approach even more general than that allowed by the theorem. For instance, Rudin [23] has given an example of a domain $D$ of type $(L)$ such that each $f \in B(D)$ has an asymptotic value along any curve which terminates at 0 and misses a suitable annular neighborhood of each boundary curve $\gamma_{k}$. It is easy to see in this case that each function in $B(D)$ has a unique asymptotic value at 0 . Of course, generally speaking, $\mathrm{Cl}(f ; 0)$ can be large; indeed, as we have noticed in $\S 5$, there exist functions $f \in B(D)$ such that $\|f\|_{\infty}=1$ and $\mathrm{Cl}(f ; 0)$ $=\{|z| \leqq 1\}$. More generally, Rudin [23] has shown that if $\zeta \in \partial R$ is not an $A B$ removable point for $R$ then there exists an $f \in B(R)$ as above; cf. [3].

The metric condition which insures the existence of $\lim _{x \rightarrow 0-} h(x), h \in B(D)$, can be strengthened to guarantee the existence of $\lim _{x \rightarrow 0-} h^{(k)}(x), k=0,1,2, \ldots, n$. In fact, one can give conditions for every derivative to extend continuously to $\{z:|z|<1, \operatorname{Re} z \leqq 0\}$. However, since the derivative of a bounded analytic function is, in general, badly unbounded (on the disc, an $H^{\infty}$ function may have a derivative of unbounded characteristic), we shall not concern ourselves with this extension. It should be noted, nonetheless, that Rudin has shown [24] that for any compact set $K$ on $\{\operatorname{Re} z=0\}$ of (linear) measure 0 there exists a domain $R$, whose boundary consists of $K$ and of circles in the open right half plane which accumulate to $K$, such that for each $f \in B(R)$ and each $k=0,1,2, \ldots, f^{(k)}(z)$ can be extended to be continuous on the closed left half plane.

See also the comments on $\S 6$.

§6. As we remarked earlier, Sarason's proof of 6.1 was somewhat different from the one we have presented. In particular, his argument relies on the F. and M. Riesz theorem and is closely related to the technique of 6.5. It seems rather remarkable that 6.1 can be obtained so simply as a consequence of the geometry of the disc.

The result of 6.3 is really quite general. If $R$ is an arbitrary plane region and if the rectifiable simple closed curve $\gamma$ is a component of $\partial R$ which lies at a positive distance from $\partial R \mid \gamma$, then the argument of 7.1 shows that each $\mu \in A(R)^{\perp}$ is absolutely continuous with respect to arc length on $\gamma$. It follows that if the (closed) boundary curves of $R$ accumulate at a countable set of degenerate boundary components $\left\{\zeta_{1}, \zeta_{2}, \ldots\right\}$ and $\mu \perp A(R)$ then $\mu=F d s+\sum c_{n} \delta_{n}$, where $\sum\left|c_{n}\right|<\infty$ and $\delta_{n}$ is the point mass at $\zeta_{n}$.

Similarly, it is a general fact for completely arbitrary $R$ that $B+Q$ is a subalgebra. It is also true that $B+Q$ is dense in $B+C$. For each point of $\partial R$ is in the closure of $R$, so that each point of $\partial R$ is a peak point for the uniform closure on $\partial R$ of rational functions analytic on $\partial R[29$, p. 52]. By Bishop's theorem [29, p. 123], $Q$ is dense in $C=C(\partial R)$.

The proof of 6.5 depends strongly on the fact that $B$ is the weak * closure of $A$, and this fact is available to us only if $\partial R$ clusters at finitely many point components of the boundary. However, if we assume the weak * density of $A$ in $B$, the final part of the proof remains valid even if the curves of $\partial R$ are allowed to cluster at a 
countable number of point components of $\partial R$. The crucial observation here is that if $\zeta_{0}$ is a boundary component which is not a peak point then there exists a positive function $K \in L^{1}(d s)$ such that $\int K d s=1$ and $\int K g d s=g\left(\zeta_{0}\right)$ for each $g \in A$. Indeed, by a theorem of Bishop [29, p. 8] there exists on $\partial R$ a probability measure $\nu$, whose mass is carried entirely on the peak points for $A$, such that $\int g d \nu=g\left(\zeta_{0}\right)$, $g \in A$. Clearly, $\nu$ contains no point masses. Now $\left(\zeta-\zeta_{0}\right) d \nu \perp A$, so by 6.3 we have $\left(\zeta-\zeta_{0}\right) d \nu \ll d s$. Thus $d v \ll d s$, i.e., $d \nu=K d s$, as required. Since $A$ is (assumed to be) weak * dense in $B$, integration against $K$ extends to a complex homomorphism (in $M_{\zeta_{0}}$ ) of $B$. The remainder of the proof of 6.5 can be carried through along the lines of the proof in $\S 6$. We omit the details.

8. Bibliography. Relatively little has been written about bounded analytic functions on general (infinitely connected) domains. There is, of course, the basic paper of Havinson [15], which is concerned, in part, with extremal problems for bounded analytic functions. Quite recently, Fisher [10] simplified some of Havinson's results. While the approaches of both these authors are rather general, the problems they consider do not, for the most part, bear directly on the questions we have studied above. One notable exception is the theorem of Fisher which says, in particular, that each function in the unit ball of $B(D)$ can be approximated pointwise boundedly on $D$ by inner functions in $B(D)$; this extends a classical result of Carathéodory. In a somewhat different direction, Rubel and Shields [21] have studied spaces of bounded analytic functions under topologies different from that induced by the uniform norm.

We have mentioned Rudin's papers [23], [24] and Beck's note [3] above; to these we should add the paper of Gamelin and Rossi [12] (or, better, [13]), the primary concern of which is the study of harmonic functions on domains of infinite connectivity. In fact, certain examples in [13] actually provided the inspiration for the results of $\S 5$; only later did we become acquainted with [23] and [24]. It is Fisher's work [8], [9], however, that seems most closely related to our results. In particular, [8] contains a proof that $A(\bar{D})$ is pointwise boundedly dense in $B(D)$ in case $D$ satisfies certain (rather restrictive) metric criteria. Part of the motivation for [8] came from Voichick's paper [27], which characterized the extreme points of $B(R)$ for a special class of infinitely connected domains (Blaschke domains).

Finally, let us note that Gamelin's approach to the results of $\$ 3$ (cf. $\$ 7)$, utilizing the techniques of Vitushkin, will appear in extenso in [11].

9. Additional remarks. Since this paper was written, new information on bounded analytic functions on infinitely connected domains has become available.

For results on pointwise bounded approximation of bounded analytic functions see [30].

The obvious analogue of Proposition 4.4 is true for an arbitrary plane domain. This observation is due to T. W. Gamelin. The simple proof is an amalgam of the argument of 4.4 with the Vitushkin technique exhibited in 2.4. 
In a recent U.C.L.A. thesis, Michael Behrens has settled special cases of several of the problems left open here. In particular, he has proved the corona conjecture for domains of type $(L)$ which satisfy $\sum r_{n} / x_{n}<\infty$ and $x_{n+1} / x_{n} \leqq c<1$ and has succeeded in describing the intersection of $M_{0}$ with the part containing $D$ in this and other cases. His techniques, which are related to the nonstandard analysis of Robinson [31], consist of an ingenious reduction of problems on $D$ to known results on the disc.

Added in proof. Behrens' work will appear in [32]. Gamelin [33] has recently shown that the corona problem on an arbitrary domain is local; this enables him to exhibit a large class of infinitely connected domains for which the corona conjecture is true. The results of $\S 5$ have just been generalized to arbitrary domains in a beautiful paper by Gamelin and Garnett [34].

\section{REFERENCES}

1. P. R. Ahern and Donald Sarason, On some hypo-Dirichlet algebras of analytic functions, Amer. J. Math. 89 (1967), 932-941.

2. Richard Arens, The maximal ideals of certain function algebras, Pacific J. Math. 8 (1958), 641-648.

3. Anatole Beck, $A$ theorem on maximum modulus, Proc. Amer. Math. Soc. 15 (1964), 345-349.

4. E. A. Bishop, Abstract dual extremal problems, Notices Amer. Math. Soc. 12 (1965), 123.

5. Lennart Carleson, Interpolations by bounded analytic functions and the corona problem, Ann. of Math. (2) 76 (1962), 547-559.

6. E. F. Collingwood and A. J. Lohwater, The theory of cluster sets, Cambridge Univ. Press, Cambridge, 1966.

7. Nelson Dunford and Jacob T. Schwartz, Linear operators. Part I, Interscience, New York, 1966.

8. Stephen D. Fisher, Bounded approximation by rational functions, Pacific J. Math. 28 (1969), 319-326.

9. - Rational functions, $H^{\infty}$, and $H^{p}$ on infinitely connected domains, Illinois J. Math. 12 (1968), 513-523.

10. - On Schwarz's lemma and inner functions, Trans. Amer. Math. Soc. 138 (1969), 229-240.

11. T. W. Gamelin, Uniform algebras, Prentice-Hall, Englewood Cliffs, N. J., 1969.

12. T. W. Gamelin and Hugo Rossi, "Jensen measures and algebras of analytic functions", in Function algebras, edited by F. Birtel, Scott, Foresman and Co., Chicago, Ill., 1966, pp. 15-35.

13. - Jensen measures and algebras of analytic functions, preprint version of [12].

14. Irving Glicksberg, Measures orthogonal to algebras and sets of antisymmetry, Trans. Amer. Math. Soc. 105 (1962), 415-435.

15. S. Ja. Havinson, Analytic capacity of sets, joint nontriviality of various classes of analytic functions, and the Schwarz lemma in arbitrary domains, Amer. Math. Soc. Transl. (2) 43 (1964), 215-266.

16. Henry Helson and Donald Sarason, Past and future, Math. Scand. 21 (1967), 5-16.

17. Kenneth Hoffman, Banach spaces of analytic functions, Prentice-Hall, Englewood Cliffs, N. J., 1962. 
18. Kenneth Hoffman, Bounded analytic functions and Gleason parts, Ann. of Math. (2) 86 (1967), 74-111.

19. Kenneth Hoffman and Hugo Rossi, Extensions of positive weak *-continuous functionals, Duke Math. J. 34 (1967), 453-466.

20. Charles E. Rickart, General theory of Banach algebras, Van Nostrand, Princeton, N. J., 1960.

21. L. A. Rubel and A. L. Shields, The space of bounded analytic functions on a region, Ann. Inst. Fourier (Grenoble) 16 (1966), 235-277.

22. Walter Rudin, Analytic functions of class $H_{p}$, Trans. Amer. Math. Soc. 78 (1955), 46-66.

23. - Some theorems on bounded analytic functions, Trans. Amer. Math. Soc. 78 (1955), 333-342.

24. —- Essential boundary points, Bull. Amer. Math. Soc. 70 (1964), 321-324.

25. Donald Sarason, Generalized interpolation in $H^{\infty}$, Trans. Amer. Math. Soc. 127 (1967), 179-203.

26. M. Tsuji, Potential theory in modern function theory, Maruzen, Tokyo, 1959.

27. Michael Voichick, Extreme points of bounded analytic functions on infinitely connected regions, Proc. Amer. Math. Soc. 17 (1966), 1366-1369.

28. Max L. Weiss, Cluster sets of bounded analytic functions from a Banach algebraic viewpoint, Ann. Acad. Sci. Fenn. Ser. A I 367 (1965).

29. Lawrence Zalcman, Analytic capacity and rational approximation, Springer-Verlag, Berlin, 1968.

30. T. W. Gamelin and John Garnett, Constructive techniques in rational approximation, Trans. Amer. Math. Soc. (to appear).

31. Abraham Robinson, Non-standard analysis, North-Holland, Amsterdam, 1966.

32. Michael Behrens, The corona conjecture for a class of infinitely connected domains, Bull. Amer. Math. Soc. (to appear).

33. T. W. Gamelin, Localization of the corona problem, Pacific J. Math. (to appear).

34. T. W. Gamelin and John Garnett, Distinguished homomorphisms and fiber algebras, Amer. J. Math. (to appear).

MassachusetTs INSTITUTE OF TechNology, Cambridge, MassachusetTs

STANFORD UNIVERSITY, Stanford, California 